\title{
Stellar populations in HII galaxies ${ }^{\star}$
}

\author{
P. Westera ${ }^{1}$, F. Cuisinier ${ }^{1}$, E. Telles ${ }^{2}$, and C. Kehrig ${ }^{2}$ \\ 1 GEMAC, Observatório do Valongo/UFRJ, Ladeira do Pedro Antônio, 43, 20.080-090 Rio de Janeiro, Brazil \\ e-mail: [westera; francois] @ov.ufrj.br \\ 2 Observatório Nacional, Rua José Cristino, 77, 20.921-400 Rio de Janeiro, Brazil \\ e-mail: [etelles;kehrig]@on.br
}

Received 4 April 2003 / Accepted 5 May 2004

\begin{abstract}
We analyse the stellar content of a large number of HII galaxies from the continua and absorption features of their spectra using population synthesis methods, in order to gain information about the star formation histories of these objects. We find that all galaxies of our sample contain an old stellar population ( $\geq 1 \mathrm{Gyr}$ ) that dominates the stellar mass, and in a majority of these we also found evidence for an intermediate-age population $\geq 50 \mathrm{Myr}$ apart from the presently bursting, ionizing young generation $\leq 10^{7} \mathrm{yr}$.
\end{abstract}

Key words. catalogs - stars: atmospheres - HII regions - galaxies: evolution - galaxies: starburst - galaxies: stellar content

\section{Introduction}

HII galaxies are easily recognised from their prominent emission line spectra, very similar to those of HII regions (recombination lines of hydrogen and helium, as well as forbidden lines of elements like oxygen, neon, nitrogen, sulphur, and others, mainly in their first and second ionization stages). The continuum part of their spectra in the visible is much fainter, and mostly of stellar origin. HII galaxies show very intense star formation, which is responsible for the ionization of the gas and the subsequent emission lines. In most cases, HII galaxies possess old populations as well, that can generally be detected at a reasonable distance from their centers (Telles \& Terlevich 1997).

For dwarf galaxies, which constitute a significant fraction of HII galaxies, the gas content is however too low for the star formation rate to have been sustained at the present level during their whole life. It is generally believed (e.g. Searle et al. 1973) that they spend most of their lives in a quiescent phase, and that the intense star formation phases in which they are encountered now are very short. The old population that is generally detected would then be the result of the accumulation of former bursts. Some exceptions may exist, like IZw18, where some authors say that the young bursting population is the only one (e.g. Papaderos et al. 2002), although others argue that a faint old underlying population does indeed exist, only detectable in the outskirts of the galaxy (e.g. Östlin 2000; Aloisi et al. 1999), and may be the result of an early low level continuous phase of star formation, as proposed by Legrand et al. (2000).

The bursting mechanisms are still unknown, although various have been proposed, of internal - (e.g. interaction of hot

\footnotetext{
* Appendix A is only available in electronic form at
} http://www.edpsciences.org and cold gas phases, like in Tenorio-Tagle et al. 1999; see also Telles \& Terlevich 1995) or external origin (e.g. tidal forces Salzer 1989; ram pressure Murakami \& Babul 1999). The burst pattern is unclear as well: Does star formation occur simultaneously over the whole extent of the galaxy, or is the present star forming region formed by the accumulation of successive smaller star forming events, spread over the galaxy, over a short timescale?

Although stellar population synthesis analyses cannot give any direct answer to the question of the origin of the bursts, they can be quite helpful in bringing some insight in the recent star formation history of these galaxies, and to a lesser extent in the older one, and thus contribute to answering our second question, e.g. what is the bursting pattern of HII galaxies? On this subject, we already have some evidence that the recent star formation is synchronised on scales of hundreds of parsecs rather than self-propagated. This is what Telles et al. found from a spatial-temporal analysis of optical and infrared surface photometry of a sample of HII galaxies (Telles 2002; Telles et al. 2004). It can also be seen from the $\mathrm{H} \alpha$ imaging atlas of blue compact dwarfs of Gil de Paz et al. (2003). Furthermore, stellar population synthesis allows us to put into evidence old populations (e.g. older than $1 \mathrm{Gyr}$ ), although their exact characteristics can be difficult to determine because of the mixture with much younger populations.

In this paper, we use the continuum spectra of a large and homogeneous sample of HII galaxies to gain information about their (star formation) histories. Similar studies include the work done by Raimann et al. (1996), Cid Fernandes et al. (2003), and Kong et al. (2003). Raimann et al. (1996) use a star cluster spectral base to identify the stellar populations present in their sample. They find that HII galaxies are typically age-composite stellar systems, presenting important contributions from 
generations up to as old as $500 \mathrm{Myr}$, and they detect a significant contribution of populations with ages older than $1 \mathrm{Gyr}$ in two groups of HII galaxies. Cid Fernandes et al. use three absorption line strength indices and two continuum colours to place the spectra in an evolutionary diagram, whose axes carry the contribution of a young ionizing $(\leq 10 \mathrm{Myr})$, an intermediate (100 Myr), and an old ( $\geq 1 \mathrm{Gyr}$ ) population to the total flux, as determined by comparing their indices with the same indices in synthetic spectra of these ages. They also find evidence for populations of all three age groups. With the same algorithm as Cid Fernandes et al., but using more absorption lines and the continuum fluxes at five wavelengths and a base of 35 star-cluster spectra by Bica (1988), Kong et al. (2003) find that blue compact galaxies (many of which are HII galaxies) are typically age-composite stellar systems with stars from all three age groups contributing significantly to the $5870 \AA$ continuum emission of most galaxies in their sample.

In this work we follow a similar approach: we define six spectral indices and determine for each spectrum the combination of the synthetic spectra of an old - and a young/intermediate stellar population, for which the indices of the empirical spectrum are best reproduced. The synthetic spectra are produced by two widely used evolutionary synthesis codes, GISSEL (Charlot \& Bruzual 1991; Bruzual \& Charlot 1993, 2000) and STARBURST (Leitherer et al. 1999), one of which (GISSEL) implements the new BaSeL 3.1 stellar spectral library, (Basel standard Stellar Library 3.1) (Westera et al. 2002; Westera 2001), which was calibrated using photometric data of globular cluster stars to improve the reproduction of the continua of the spectra of low metallicity stars. The goal of performing the analysis with two different libraries was on the one hand to check the reliability of the results, and on the other hand to find out through comparison of the results if the BaSeL 3.1 stellar library is able to reproduce the spectra of stars and populations beyond the parameter range of the objects used for its calibration, e.g. if the library can also be used for young stars and populations.

The layout of this article is the following: in Sect. 2, the catalog of spectra analysed in this work is presented. Section 3 gives a detailed description of the method we used to analyse the spectra. In Sect. 4, we discuss the accuracy of the method and its ability to give meaningful results. The main results are discussed in Sect. 5. A summary and the main conclusions can be found in Sect. 6 .

\section{The spectra}

The data base of this work consists of a catalog of intermediate resolution $(R \sim 1000)$ HII galaxy spectra in the range of 3700 to $7500 \AA$. It contains about 200 HII galaxy spectra observed with high signal-to-noise at the $1.52 \mathrm{~m}$ telescope at the European Southern Observatory (ESO) within the agreement between Brazil and ESO. All spectra were observed with the same instrumentation and reduced in a homogeneous way.

The apertures were centered on star forming knots, and our work thus presents a bias towards young populations (as a matter of fact like all the HII galaxies spectroscopic surveys made up to now). The apertures we chose had the size of a few typical

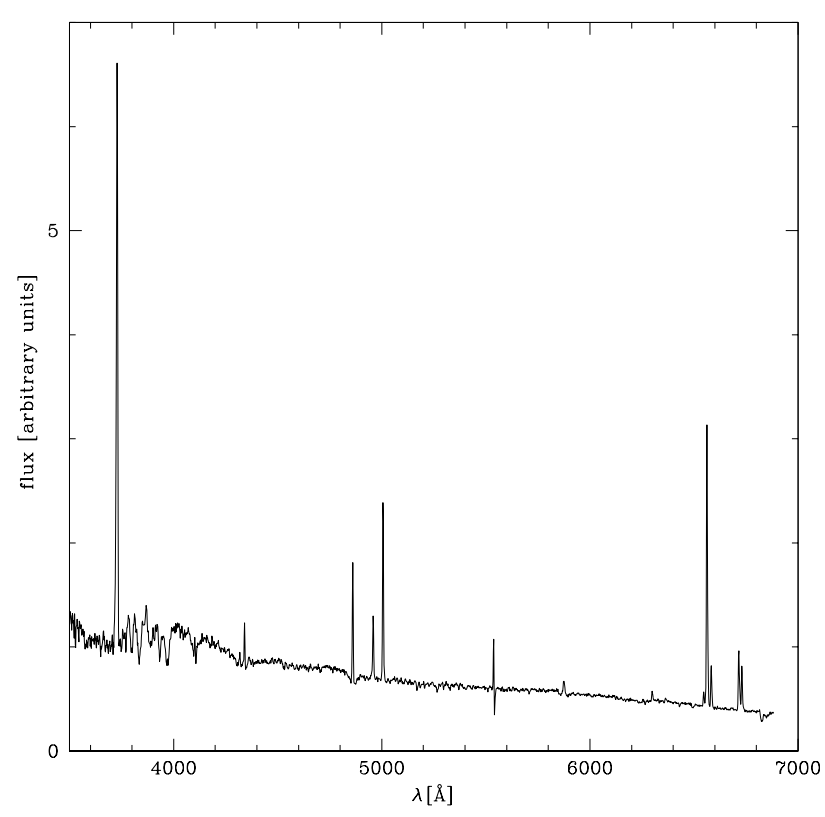

Fig. 1. Example of an HII galaxy spectrum (UM 69, taken on August, the 18th, 1998).

seeing widths, generally encompassing several stellar clusters. We therefore do not expect to have pure single stellar populations, but rather local mixes. Another important point is that a significant fraction of the galaxies were observed in several apertures, so we do not get only the most prominent knot, but also secondary ones (contrary to other spectroscopic studies), where the young population(s) is (are) less dominant in the integrated light. Table A.1 gives our galaxy sample and measured spectral indices as described below. Column 1 lists the names of the spectra, consisting of the names of the galaxies, and, in those cases where the apertures were centered on a secondary knot, an indication between brackets about the position of the aperture, where "Cent" stands for the centre of the galaxy, and the abbreviations "N", "E", "S", and "W" (and "NE" etc.) indicate that the aperture was positioned at knots at the North, East, South or West of the galaxy. Column 2 gives the type of the spectra ("in": integrated spectra, "re": spectra of individual regions). For more details about the catalog, see Kehrig et al. (2004).

The spectra were corrected for redshift, and then for internal gas extinction. The latter was done by converting the absorption constants $C_{\mathrm{H} \beta}$ as determined by Kehrig et al. (2004) into extinction constants $E(B-V)_{\text {gas }}$ using $E(B-V)_{\text {gas }}=$ $0.665 \times C_{\mathrm{H} \beta}$ (Sampson 2003), then correcting for systematic differential extinction between the stellar populations and the gas employing $E(B-V)_{\text {cont }}=0.44 \times E(B-V)_{\text {gas }}($ Calzetti et al. 2000) (which leads to $\left.E(B-V)_{\text {cont }}=0.2926 \times C_{\mathrm{H} \beta}\right)$, and finally dereddening the spectra using the extinction law of Fluks et al. (1994).

Figure 1 shows a typical corrected spectrum. Apart from the typical emission lines of an HII region, most spectra also show significant continuum contributions from a mixture of stellar populations. A closer examination of this continuum reveals that many of these spectra show signatures of young/intermediate populations (certain absorption lines, 
i.e. of hydrogen, and a blue continuum), as well as spectral properties of old populations (a $4000 \AA$ break, and absorption lines of heavy elements like calcium, iron, and magnesium), which leads to the conclusion that many of the galaxies of the sample have undergone periods of star formation before the present ionizing massive stellar population. The goal of this work is to analyse the stellar content of these galaxies, and gain information about their star formation histories, using the continua and the most evident absorption features of their spectra.

\section{Method}

We wish to characterise the population mixture in our galaxies using the sensitivity of their spectra to the properties of these populations, e.g. abundances - or metallicity - and ages. Although we could directly fit modeled spectra to the observed ones, and evaluate the various population parameters (e.g. ages and metallicities) as the ones corresponding to the "best-fitting" model spectrum, we prefer to proceed in a more conservative way, and use a set of pre-defined indices, chosen for their sensitivity to the parameters we want to derive. The fitting procedure is thus done to the indices, and not to the raw spectra. This presents the advantage of emphasising only useful features in the spectra, giving a zero weight to parts that either are badly flux calibrated (e.g. wavelengths lower than $\lambda 3900 \AA$ ), are contaminated by emission lines from the gas, or are very noisy, or simply provide redundant information. Because of the finite (and relatively low) number of indices, the maximum number of free parameters we adopt to characterise our galaxies is constrained in a straightforward manner.

We define six indices, relatively independent from each other, quantifying certain properties of the spectra, which are particularly suitable for identifying and characterising the stellar populations present in the galaxies (the shape of the continuum, the $4000 \AA$ break $\mathrm{D}(4000)$, and certain spectral (absorption) line strength indices). Most of the indices are inspired by the indices defined by Worthey et al. (Worthey et al. 1994; Worthey 1994) and carry the same names, and by analogy are defined in the following way:

Mag $=-2.5 \log \left(F_{-} / F_{+}\right)$

whereas:

$F_{X}=\frac{1}{X} \int_{X} F_{\lambda} \mathrm{d} \lambda$

Note that a wavelength range $X$ can consist of several disjoint wavelength ranges (i.e. the bands to the left and right of a spectral line). The wavelength ranges of the six indices are given in Table 1, and illustrated in Fig. 2.

The continuum index is something like the $B-R$ colour with rectangular passbands. For some spectra, it was necessary to cut out a few tens of A due to contamination of the spectra by telluric skylines. For the other indices, the wavelength ranges may differ slightly from the ranges commonly used, since we optimised them for the spectra of our sample. The bands of the $4000 \AA$ break were reduced to a width of $50 \AA$ each because of the calibration uncertainties of the spectra downwards of $\lambda \sim 3900 \AA$ affecting the - band and the presence of the
Table 1. Wavelength ranges of the positive - and negative bands of the indices, with the associated errors.

\begin{tabular}{llll}
\hline \hline Index & + Range $[\AA]$ & - Range $[\AA]$ & $\sigma_{\text {index }}$ \\
\hline Continuum index & $6320-6520$ & $4500-4800$ & 0.23 \\
& $6600-6700$ & & \\
$4000 \AA$ 政ak & $4010-4060$ & $3900-3950$ & 0.03 \\
$\mathrm{H}+\mathrm{K}(\mathrm{Ca})$ & $3905-3920$ & $3923-3942$ & 0.05 \\
& $3942.5-3956$ & & \\
$\mathrm{H} \delta$ & $4055-4072$ & $4073-4095.25$ & 0.04 \\
& $4120-4145$ & $4106.5-4119$ & \\
$\mathrm{Fe} 4531$ & $4498-4517$ & $4521-4559.5$ & 0.04 \\
& $4560-4573$ & & \\
$\mathrm{Mg}_{b}$ & $5153-5161.5$ & $5162-5193.5$ & 0.05 \\
& $5194-5203.5$ & & \\
\hline
\end{tabular}

strong $\mathrm{H} \delta$ emission line within the + band of the traditional definition of this index. Of the $\mathrm{H} \delta$ line, only the wings are used, because the region around the central wavelength of this line is dominated by the emission line of the gas. The line strength indices are defined such that absorption goes to positive values and emission to negative ones. Since all our indices are absorption features, they are in general positive. However, as we do not make any correction for the inclination of the continuum, the wavelength localisation of the various bandpasses may sometimes induce negative values, as is for instance the case for $\mathrm{H} \delta$. We preferred not to make such corrections and to use indices instead of equivalent widths, because we prefer to use quantities that are more directly linked to the observed spectra, with a minimum number of in-between steps. The calculated indices of individual spectra are listed in Table A.1.

The average signal-to-noise of the spectra amounts to 12.8 at the level of the continuum around $5500 \AA$ (for the signal-to-noise values of individual spectra, see Kehrig et al. 2004). Using the relation that the error in the average flux in a passband amounts to the error per pixel divided by the square root of the number of pixels, we can calculate the typical observational errors of the narrow-band indices, whereas the typical error of the continuum index is dominated by uncertainties in the absolute flux calibration. Following Cuisinier et al. (1996) and Kong et al. (2003), we adopt a value of $10 \%$ for these uncertainties. We list the typical observational error of each index in Table 1. They are also shown as error bars in Figs. 3 and 4.

Figure 3 shows examples of how the spectra are distributed in two-index planes compared to the same indices calculated for simple stellar populations (SSPs) of a wide range of ages (dashed lines) and metallicities (solid lines) from the "BC99" ("Bruzual \& Charlot 99") SSP library which will be described later on in this section. It can be seen that the observed indices (crosses) of the spectra lie in the region where the SSPs of young and intermediate age can be found (denoted by "young" in Fig. 3), which shows that young and intermediate age populations dominate the spectra (but not necessarily the stellar mass of the galaxies). In the panels showing the continuum index vs. H $\delta$ plane (lower left), and the panel showing the $4000 \AA$ break vs. $\mathrm{H} \delta$ plane (lower right), it is striking that the observed indices of many spectra lie outside the ranges occupied by the SSP models, e.g. inside the loop in these ranges, another 




Fig. 2. Illustration of the six indices using UM 69 (the spectrum shown in Fig. 1) as an example. The dashed lines show the limits of the bands. The bands that enter with a positive sign into the calculation of the respective index are shaded with dotted lines running from the upper left to the lower right, and the bands that have a negative sign are shaded with dotted lines running from the upper right to the lower left. For a more detailed description of the indices, see the text.

indication that one single population alone cannot reproduce the sample spectra adequately.

An indication that this can be remedied by combining populations of different ages and metallicities can be seen in Fig. 4. It shows the same two-index planes as in Fig. 3 (with different scales), but here the empirical indices are compared with composite stellar populations. The composite populations are made up of an old population with $[\mathrm{Fe} / \mathrm{H}]=-1.5$ and an age of $5 \mathrm{Gyr}$ and, and a young or intermediate one with $[\mathrm{Fe} / \mathrm{H}]=-1$ and different ages ranging from 1 to $500 \mathrm{Myr}$ (different dashed lines, increasing line thickness means increasing age). The solid lines mean different mass ratios between the two populations ranging from $M_{y+i}: M_{\mathrm{o}}=0: 1$ (only an old population) to 1:0 (only a young or intermediate population), increasing line thickness means decreasing $M_{y+i}: M_{0}$. The main problem of single stellar population models to explain the distribution of the observed data in the two-indice planes is that for index combinations containing $\langle\mathrm{H} \delta\rangle$, the observed data fill a region where no models are present, within a loop created in the models at young ages (see Fig. 3, lower panels). Combining young+intermediate and old populations in composite models makes it possible to resolve this issue, filling in the loop, and thus better reproducing the combination of indices of the sample spectra than SSP models are able to do (see Fig. 4, lower panels). For these reasons, we decided to explore the stellar composition of these galaxies by assuming them to be made up of two stellar populations, an old, metal-poor one, hereafter the "old population", and a younger one (that is, of young and/or intermediate age), which for simplicity will be referred to as the "young+intermediate population".

This is a simplification, for the young+intermediate population will always contain the very young ionizing massive stellar population (age $<10 \mathrm{Myr}$, possibly mixed up with an intermediate age population, which may consist of several subpopulations (that we are unable to discern)). The old 



Fig. 3. Distribution of the sample spectra (symbols) in different two-index planes compared with SSPs (lines). The solid lines represent the evolutionary paths of SSPs of different metallicities between $[\mathrm{Fe} / \mathrm{H}]=-2.25$ and $[\mathrm{Fe} / \mathrm{H}]=+0.35$, and the dashed lines represent the locus of SSPs of constant ages ranging from 0 to $20 \mathrm{Gyr}$. The dotted lines show the values of the indices at which SSPs of average metallicity $([\mathrm{Fe} / \mathrm{H}] \sim-1)$ reach an age of around $2 \mathrm{Gyr}$, to demarcate the ranges of young/intermediate populations and old populations. Young populations tend to be in the left part of the diagrams, old ones in the right part. The typical errors in the indices are shown as error bars in the corners of the different panels.

population may also represent a combination of several, but old, populations.

The star forming history of HII galaxies may be far more complex than what can be realistically modeled in a simple manner from their spectra. Hypotheses for star formation histories are various, as briefly mentioned in the introduction; a bursting mode followed by quiescent phases, continuous star formation but sustained at a low intensity extending far back in past, or a unique single event. Many of these scenarios, however, are indiscernible from the point of view of a spectral analysis using only integrated spectra, as was also noticed by Lilly \& Fritze-von Alvensleben (2003). We thus have to limit ourselves to an arbitrary, simpler choice of populations whose properties we believe we are able to distinguish.

Within this choice, it is not possible to determine more than only a few parameters, and the results found using only two populations already furnish interesting results about the formation histories of the sample galaxies. For the five parameters
$M_{y+i}: M_{\mathrm{o}}$ (the mass relation between the young+intermediateand the old population), age $\mathrm{y}_{y+i} / \mathrm{age}_{\mathrm{o}}$ (the ages of each population), and $[\mathrm{Fe} / \mathrm{H}]_{y+i} /[\mathrm{Fe} / \mathrm{H}]_{\mathrm{o}}$ (the respective metallicities), we took into consideration the possible values given in Table 2 . Note that only two of these parameters, $M_{y+i}: M_{\mathrm{o}}$ and age $\mathrm{e}_{y+i}$, are free (thus to be determined in this work), and the discussion about the solutions will mainly consist of interpreting the values found for these two parameters. The metallicity of the young population is given by the $[\mathrm{O} / \mathrm{H}]$ values of the gas as determined from the emission lines by Cuisinier et al. (2004) in a parallel article to this one, which deals with the emission part of the spectra, as the young population should be more or less coeval with the gas, whose metallicity is the metallicity attained now by these galaxies (Cuisinier et al. 2004; Pagel et al. 1992). In those cases where $[\mathrm{O} / \mathrm{H}]$ of the gas could not be determined, we fixed $[\mathrm{Fe} / \mathrm{H}]_{y+i}$ at -1 , which is close to the average value of the other spectra. The two parameters concerning the old population, age $\mathrm{o}_{\mathrm{o}}$ and $[\mathrm{Fe} / \mathrm{H}]_{\mathrm{o}}$, are also fixed at reasonable values for such a population ( $5 \mathrm{Gyr}$, resp. $-1.5 \mathrm{dex}$ ). However, 



Fig. 4. Distribution of the sample of spectra (symbols) in different two-index planes compared with composite stellar populations (lines). In relation to Fig. 3, the range of the indices has been centered on the region where most of the observed data lie. The solid lines represent the compositions of an old population (age $5 \mathrm{Gyr},[\mathrm{Fe} / \mathrm{H}]=-1.5)$ and a young or intermediate one $([\mathrm{Fe} / \mathrm{H}]=-1)$ with different mass ratios ranging from $M_{y+i}: M_{\mathrm{o}}=0: 1$ (only an old population) to 1:0 (only a young/intermediate population). The mass of the young population decreases with increasing line thickness, and the age of the young population varies along the lines. The dashed lines are lines of constant age of the young/intermediate population varying from 0 to $500 \mathrm{Myr}$ (increasing line thickness means increasing age), whereas the mass ratio between the two populations varies along the lines. They converge towards the point where the mass of the young/intermediate population is zero. The typical errors in the indices are shown as error bars in the corners of the different panels.

Table 2. Possible values of the population parameters.

\begin{tabular}{ll}
\hline \hline Parameter & Possible values \\
\hline$M_{y+i}: M_{\mathrm{o}}$ & $0: 1,1: 100,1: 30,1: 10,1: 3,1: 1,3: 1,10: 1,1: 0$ \\
$\mathrm{age}_{y+i}$ & $1,2,5,10,20,50,100,200,500 \mathrm{Myr}$ \\
{$[\mathrm{Fe} / \mathrm{H}]_{y+i}$} & Taken from Cuisinier et al. (2004) or -1 \\
$\mathrm{age}_{\mathrm{o}}$ & Fixed at $5 \mathrm{Gyr}$ \\
{$[\mathrm{Fe} / \mathrm{H}]_{\mathrm{o}}$} & Fixed at -1.5 \\
\hline
\end{tabular}

the analysis is not very sensitive to the exact values of these two parameters, since spectral features do not vary much in this parameter range. Note also that this approach allows solutions consisting of only an old - or only a young+intermediate population $\left(M_{y+i}: M_{\mathrm{o}}=0: 1\right.$ or $\left.1: 0\right)$.

The best fits were performed using two different libraries of SSPs in order to determine the reliability of the results.
Another by-product of this study is to test if the new synthetic stellar spectral library BaSeL 3.1, which was calibrated using globular cluster photometrical data to improve the reproduction of the continuum contribution of spectra of low metallicity stars $([\mathrm{Fe} / \mathrm{H}] \lesssim-1)$, can be used for young or intermediate stars and populations, thus for objects outside the parameter range of the objects used for its calibration. The first SSP library (hereafter the "BC99" library) was produced using the Bruzual and Charlot 2000 Galaxy Isochrone Spectral Synthesis Evolution Library (GISSEL) code (Charlot \& Bruzual 1991; Bruzual \& Charlot 1993, 2000), implementing the Padova 2000 isochrones (Girardi et al. 2000) combined with the BaSeL 3.1 "Padova 2000" stellar library. We used a Salpeter initial mass function (IMF) from 1 to $100 M_{\odot}$, to be able to make direct comparisons with the second SSP library, from now on called the "Starburst" library, which uses 


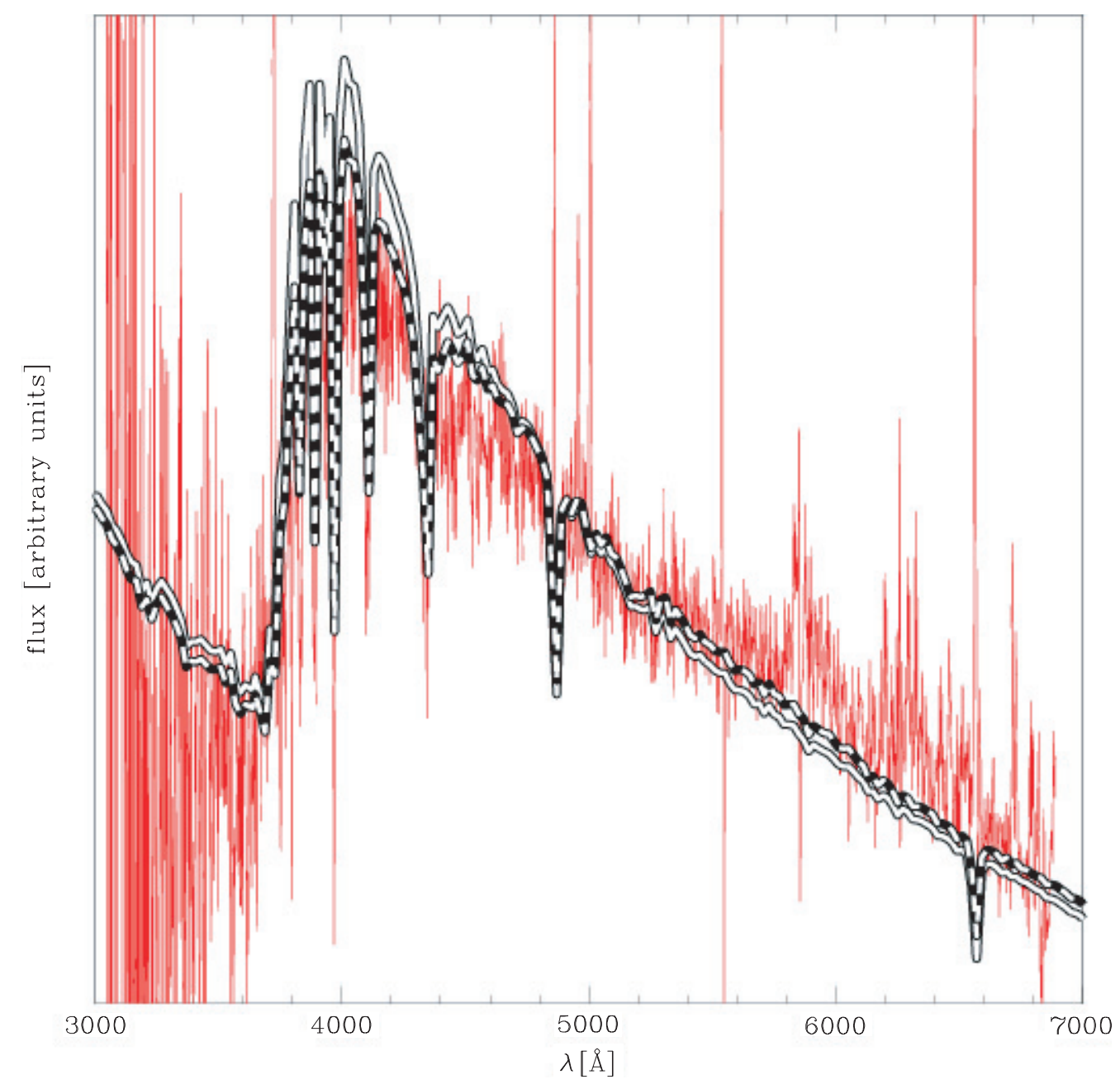

Fig. 5. Example of a "best fit". The thin line represents the empirical spectrum (UM 137(W), taken on August, the 18th, 1998), the thick solid white line shows the best fit spectrum obtained using the "BC99" library, and the thick dashed white line (which sometimes hides the solid line) shows the best fit using "Starburst".

the same IMF. The "Starburst" library consists of spectra from the STARBURST99 data package (Leitherer et al. 1999) using the option of including nebular continuum emission (Fig. 1 on the STARBURST99 web site). It implements the predecessor of the BaSeL 3.1 library, the widely used BaSeL 2.2 library (Lejeune et al. 1997, 1998), and for stars with strong mass loss the Schmutz et al. (1992) extended model atmospheres, combined with the Geneva isochrones (Meynet et al. 1994; Schaller et al. 1992; Schaerer et al. 1993a,b; Charbonnel et al. 1993). Apart from the implementation of different isochrones and stellar libraries, one of the main differences between the "BC99" and the "Starburst" libraries lies in the inclusion of the nebular continuum emission under the hypothesis of optical thickness of the nebulae, e.g. that all photons with wavelengths below $912 \AA$ are absorbed. This affects the spectra of young populations (up to $10 \mathrm{Myr}$ ) shortward of $4500 \AA$. For the old population, the "BC99" spectrum was used, since the Starburst99 data package only contains spectra up to $900 \mathrm{Myr}$.

The best fits were performed by minimising the quantity

$\chi^{2}=\sum_{\text {indices }}\left(\frac{\langle i\rangle_{\mathrm{emp}}-\langle i\rangle_{\text {synth }}}{\sigma_{\langle i\rangle}}\right)^{2}$ where $\langle i\rangle_{\mathrm{emp}}$ is the value of the $i$ th index of the empirical spectrum, $\langle i\rangle_{\text {synth }}$ is the value of the same index of the synthetic spectrum, produced by adding the synthetic spectra of the young+intermediate and the old population in the relation $M_{y+i}: M_{\mathrm{o}}$, and $\sigma_{\langle i\rangle}$ is the observational error in the $i$ th index of the spectrum. As the $\mathrm{Fe} 4531$ and $\mathrm{Mg}_{b}$ indices proved too affected by noise for our purpose, we decided not to adopt them in the fits (although the lines are clearly visible in many of the spectra, which is why they were originally included in the set). The fact that $[\mathrm{Mg} / \mathrm{Fe}]$ is probably non-solar in these galaxies may cause additional complications as well if we use these indices. However, we did include them in the analysis performed in Sects. 4.1 to 4.3 .

In the end, a selection of acceptable solutions was made. All spectra which had a too bad signal-to-noise or a strange shape, indicating calibration problems in the data reduction, were eliminated from the sample. This selection was done by eye. Figure 5 shows an example of an accepted fit.

A major concern in our method stems from the fact that the spectra of our sample have a higher resolution than the spectra in the "BC99" and "Starburst" libraries. Whereas the sample spectra have a resolving power of $R \sim 1000$, the synthetic 
spectra have only around $R \sim 250$ in the optical. However, degrading our spectra to this resolution would significantly reduce the amount of information they hold, particularly in the $\mathrm{H} \delta$ index, for which the contamination of the $\mathrm{H} \delta$ emission line would increase dramatically. As this line is in general much stronger than the absorption line which the index is trying to quantify, it would dominate the index after a degradation. Correcting for the contribution of the emission line using an estimate of the $\mathrm{H} \delta$ flux based on the fluxes of other Balmer lines would still leave us with an error as large as the error of this estimate, which is much larger than the variation of the index itself. For this reason, we decided to study the effect of resolution on the fits rather than to degrade our sample spectra. This study was done by performing the best fits on synthetic highresolution spectra of known ages. The synthetic spectra, in the following called the "BC-2000" synthetic SSP spectra, were again produced with the GISSEL software using the same input parameters (IMF, stellar evolutionary tracks) as for the "BC99" library but this time implementing the BC-2000 (Bruzual \& Charlot 2000) stellar library. This is a library of empirical stellar spectra, mainly from a catalog assembled by Pickles (1998), with higher resolution than the theoretical libraries $(R \sim 500)$, but containing only solar metallicity stars. For a more detailed description of the BC-2000 stellar library, see Bruzual $\&$ Charlot $(2000,2003)$. We then determined the ages of these spectra in the same way as we determined the population properties of the HII galaxy spectra: by minimising the $\chi^{2}$ defined in Eq. (3) (using a signal-to-noise value of 12.8 for the estimate of the errors $\sigma_{\langle i\rangle}$ of the BC-2000 spectra, which corresponds to the average signal-to-noise of the HII galaxy spectra) for "BC99" and "Starburst" SSPs of solar metallicity, and considering ages of 1, 2, 5, 10, 20, 50, 100, 200, and 500 Myr. In Fig. 6, the best fitting ages obtained in this way are shown as a function of the age of the original "BC-2000" SSP ages. In spite of the different resolutions of the "BC-2000" (input) spectra and the spectra used to fit the ages ("BC99" and "Starburst"), the ages are well reproduced with a slight tendency to favour young or intermediate ages. We conclude from this that the higher resolution of the empirical spectra does not pose greater problems for the fitting procedure and for the accuracy of the parameters we wish to determine. For these reasons, we preferred not to degrade the spectra of our sample to the "BC99" and "Starburst" resolution.

\section{Accuracy of the method}

To check how useful our indices are for deducing population mass ratios and ages from our spectra we performed various tests with the observed indices and derived parameters.

After rejection of all spectra that presented strange behaviour of the fitted model spectra, solutions were found for 123 spectra from 78 different galaxies.

Performing the best fits while varying the indices by their typical observational errors (as listed in Table 1) showed that in the worst case (if the errors in all indices conspire), they can modify the solutions by around one step (a factor of $\sim 3$ ) in $M_{y+i}: M_{\mathrm{o}}$ or two steps (a factor of $\sim 5$ ) in age ${ }_{y+i}$. For most spectra, however, the errors will not conspire, as they are random and not correlated, so the statistical properties of the sample

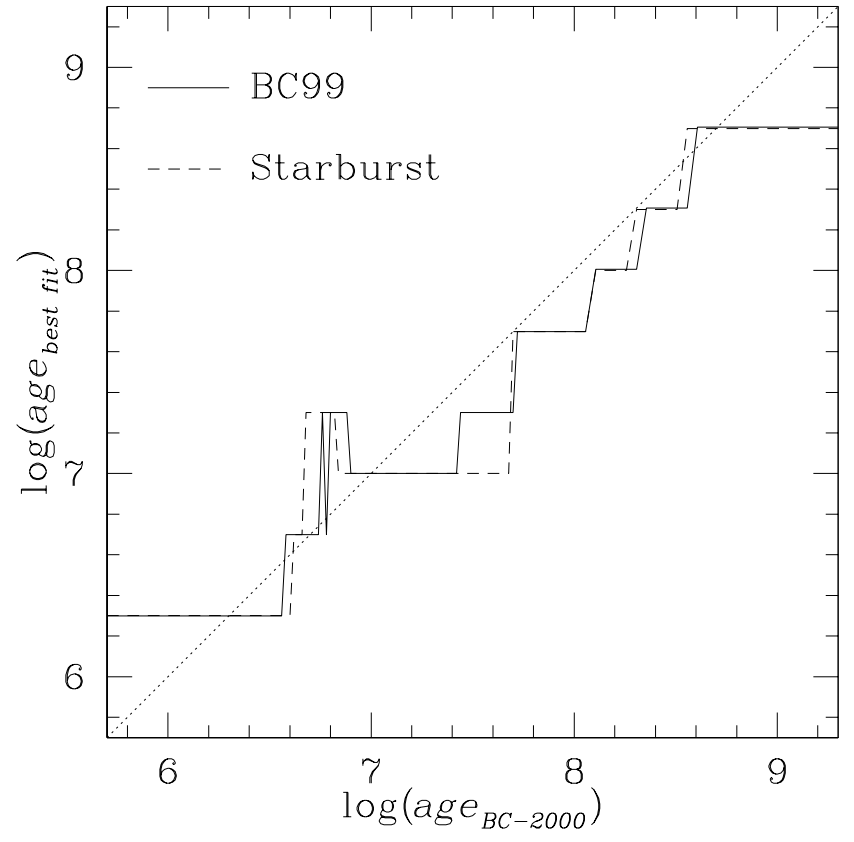

Fig. 6. Ages of the best fits performed on "BC-2000" synthetic SSPs as a function of their ages. The solid line shows the best fits using the "BC99" library, and the dashed line shows the best fits using "Starburst". The dotted line represents identity.

discussed in this section should not be too affected by the observational errors. The solutions are presented in Table A.2.

\subsection{Correlations between the empirical indices and the obtained parameters}

We will first investigate how these results depend upon the different indices of the galaxy spectra. $M_{y+i}: M_{\mathrm{o}}$ correlates (weakly) with the continuum index and with $\mathrm{D}(4000)$, but not with the other indices (not shown here). Figure 7 shows the correlations of the indices with the derived age of the young+intermediate population. It correlates with $\mathrm{D}(4000)$ (upper middle) and (weakly) with the continuum index (upper left) and $\mathrm{H} \delta$ (lower left). For the continuum index, the trend reverses at age ${ }_{y+i}=5 \mathrm{Myr}$, where the nebular emission starts to influence the continuum (for very young ages it even dominates the spectra below $3600 \AA$ ). The other indices correlate only weakly (if at all) with age $e_{y+i}$.

\subsection{Reproduction of the indices}

The examination of how well the indices of the galaxy spectra are being reproduced in the fits yields the answer that could be expected from the typical observational errors of each index (Fig. 8): the $4000 \AA$ break is well reproduced in the best fit spectra, and the continuum index a bit less well (but acceptably). The $\mathrm{H}+\mathrm{K}(\mathrm{Ca})$ and $\mathrm{H} \delta$ indices represent the worst cases of our adopted fit indices: our fit values present some correlation with the observed values, but with a systematic tendency to an underestimation of $\mathrm{H}+\mathrm{K}(\mathrm{Ca})$ and an overestimation of $\mathrm{H} \delta$. For $\mathrm{H} \delta$, this is due to the fact that the models only predict values above $\sim 0.03$. This can also be seen in the lower panels of 



Fig. 7. The ages of the young+intermediate population as a function of the indices of the spectra. The crosses represent the solutions found using "BC99", and the squares represent the "Starburst" solutions. The typical errors in the indices are shown as error bars in the upper left corners of the different panels.

Fig. 4. A possible explanation is that the underlying population in some of the galaxies could be older than $5 \mathrm{Gyr}$, which would lead to lower values of $\mathrm{H} \delta$. However, the trends in $\mathrm{H}+\mathrm{K}(\mathrm{Ca})$ and $\mathrm{H} \delta$ are unlikely to influence the statistical properties of our overall results in a systematic manner, as they drive the parameter $M_{y+i}: M_{\mathrm{o}}$ in opposite directions, as well as the age of the young+intermediate population.

\subsection{Comparison of the solutions obtained with the two different libraries}

To determine to what amount these results depend on the SSP library (and thus on the stellar library) that was used, it is necessary to compare the results obtained with the two different libraries for the same spectra. Figure 9 shows the agreement between the best fits made with the two libraries for the $M_{y+i}: M_{\mathrm{o}}$ ratio, and Fig. 10 shows the same for the age $_{y+i}$. In most cases, the two libraries yield the same value for $M_{y+i}: M_{\mathrm{o}}$, and this parameter differs significantly between the two libraries only in three cases. In these three cases, either the "BC99" - or the "Starburst" solution predicts the existence of only a young+intermediate population $\left(M_{y+i}: M_{\mathrm{o}}=1: 0\right)$, whereas the other library predicts a significant old population $\left(M_{y+i}: M_{\mathrm{o}}=1: 1\right.$ or $\left.1: 30\right)$. A closer look at these spectra does not reveal why the fits with the two libraries yield such different results. In spite of the completely different population parameters, the best fitting composite model spectra of the "BC99" and "Starburst" solutions look very similar. In almost half of 


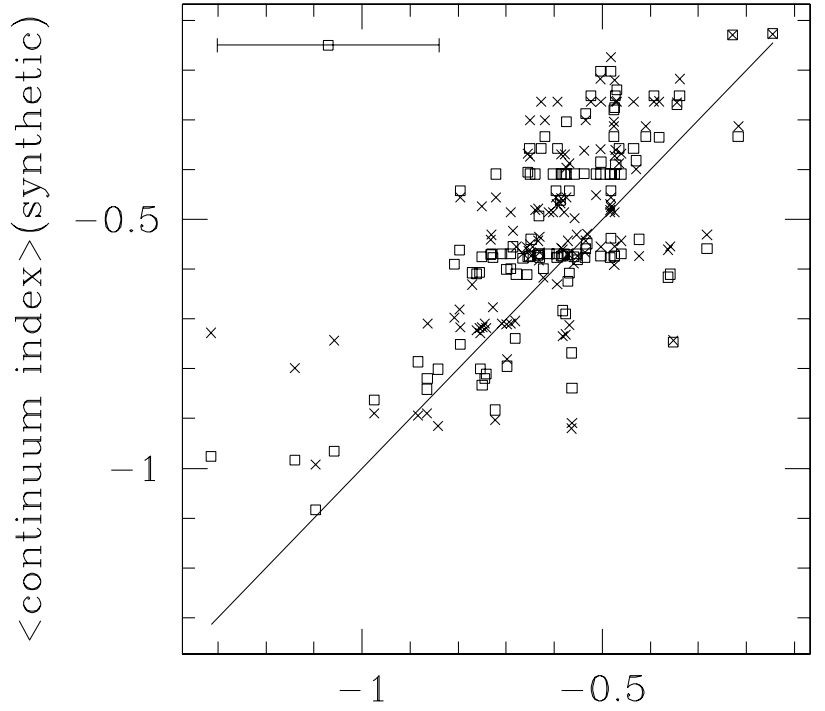

$<$ continuum index $>$ (observed)

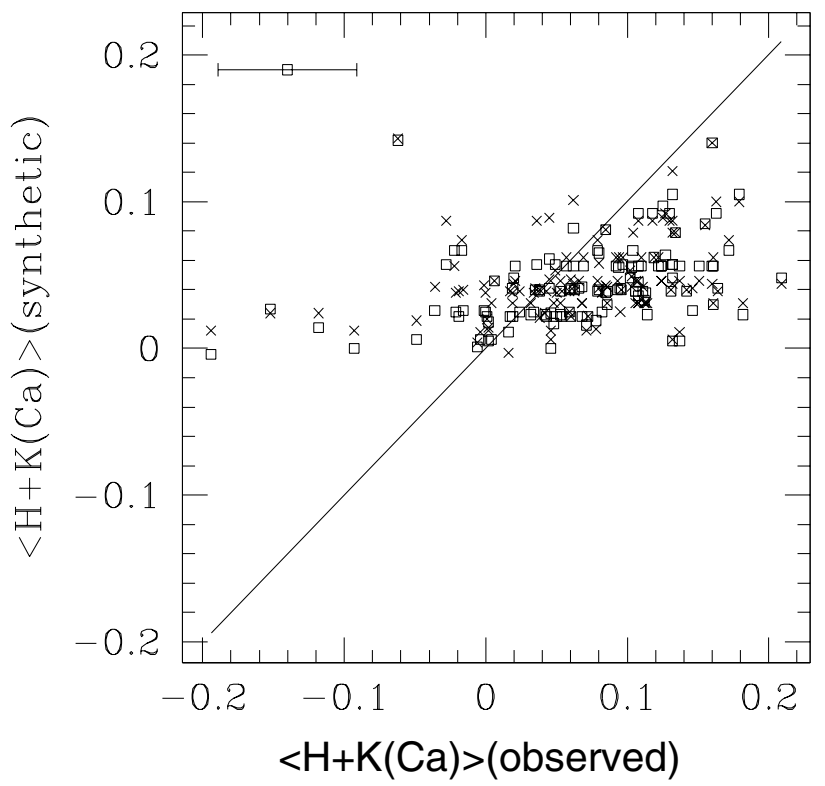

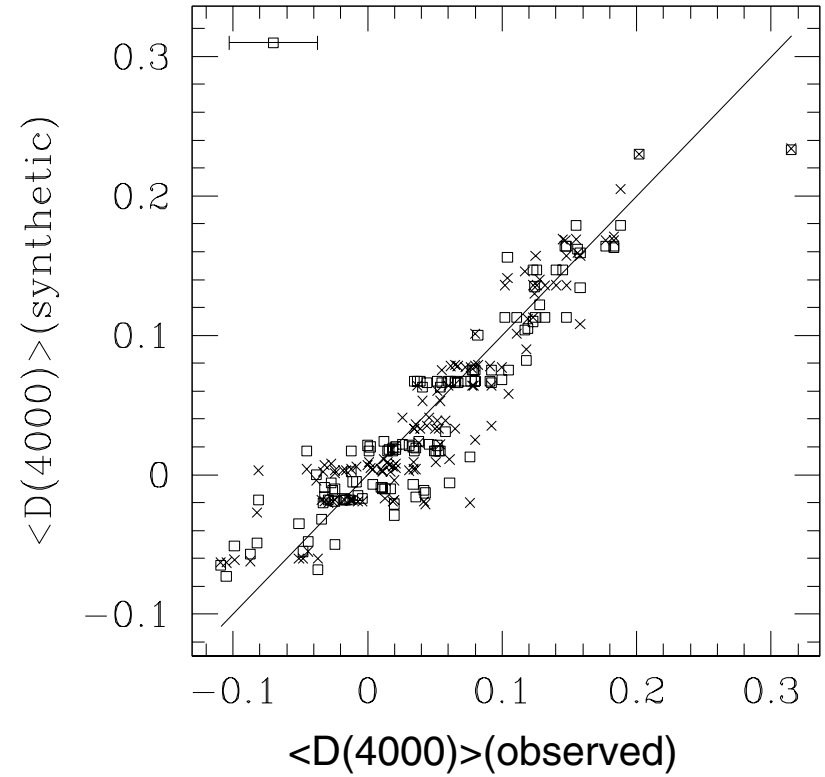



Fig. 8. The indices of the empirical spectra vs. the same indices of the synthetic spectra (thus the reproduction of these indices). The crosses represent the solutions found using "BC99", and the squares represent the "Starburst" solutions. The lines represent identity (perfect reproduction). The typical errors in the observed indices are shown as error bars in the upper left corners of the different panels.

the cases however, the two libraries yield identical results, and in most of the rest the solutions are similar enough. The two libraries also do not show any systematic differences in the indices calculated from the best fit spectra (see Fig. 11).

The similarities between the solutions found with the two libraries are also confirmed when comparing the $\chi^{2}$ (Fig. 12).

Thus, for the vast majority of the spectra, the solutions found with both libraries show good agreement in all three: the population parameters found, the reproduction of the indices, and $\chi^{2}$, which gives us confidence in the solutions and in our general conclusions. Given all these similarities, it is not possible to conclude from this study alone if the BaSeL 3.1 stellar library does a better job at reproducing spectra than its predecessor. It is encouraging that the two SSP libraries yield results of comparable quality, even though the "Starburst" library has additional features implemented (nebular continuum emission, inclusion of the Schmutz model atmospheres for stars with strong mass loss) aimed at a better reproduction of the spectra of young+intermediate populations. Further studies, aimed more directly at the properties for which the BaSeL 3.1 library was designed (a better reproduction of the continuum contribution of low metallicity stellar spectra) will be necessary to clarify this point.

\section{Results and discussion}

The composition of HII galaxies may be far more complex than just the two young+intermediate and old populations we are 


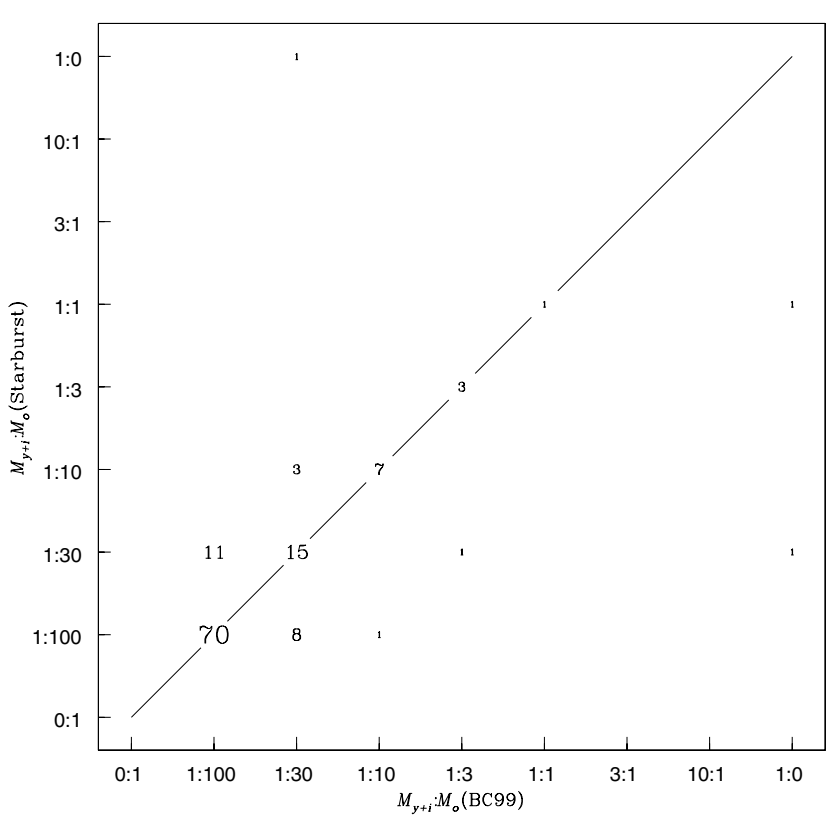

Fig. 9. The number of cases, in which a certain value $(x)$ of $M_{y+i}: M_{\mathrm{o}}$ was found using "BC99" and another $(y)$ value was found using "Starburst".

using in our modeling - as we stated earlier, they can have a far more complicated star forming history, not only in time but also in its spatial distribution. Our modeling represents our lack of ability to segregate more than only a few stellar populations with very distinct ages. It is in particular very hard to differentiate old populations because their spectrophotometric properties are so similar (Lilly \& Fritze-von Alvensleben 2003); that is the reason why we grouped them all in a generic old population, but we have to keep in mind that we have very little insight in the constitution of this generic old population.

Nevertheless, some star forming history scenarios can clearly be ruled out from the point of view of our modeling, such as extreme limiting scenarios presenting one single population, either only old or only young.

In this section, we will discuss various possible star forming histories in the light of our modeling.

\subsection{Mass ratios of the young+intermediate and old populations}

We were able to derive mass ratios between the young+intermediate and the old populations. Figure 13 shows how these mass ratios are distributed. The upper panel is a histogram of the best fitting $M_{y+i}: M_{\mathrm{o}}$ of all apertures for all galaxies for which solutions were found, and the lower panel is a histogram made up of one $M_{y+i}: M_{\mathrm{o}}$ value (the lowest one found) per galaxy, in order to emphasise the old stellar component.

Our study indicates that in terms of mass, the old population is overwhelmingly dominant, by a factor of $\simeq 100$ in most of the apertures in all galaxies (the peaks at $M_{y+i}: M_{\mathrm{o}}=1: 100$ of Fig. 13).

For the one galaxy where the integrated spectrum yielded only a young+intermediate population (DDO155) we were



Fig. 10. The number of cases, in which a certain $(x)$ value of age $e_{y+i}$ was found using "BC99" and another $(y)$ value was found using "Starburst".

able to obtain evidence for an old population in other apertures. Only in two cases that were observed in one aperture only (Tol1223-359 and UM 323) did the best fits yield no old population (the sole points at $M_{y+i}: M_{\mathrm{o}}=1: 0$ of panel $\mathrm{b}$ of Fig. 13), albeit only in the fit using one of the two libraries. In these cases, the fit with the other library did present a (dominating) old population (see also Sect. 4.3), so even in these dubious cases we have some evidence for an old population.

It is however difficult to make any further statement on the nature of the old population, except that it should be older than $\simeq 1 \mathrm{Gyr}$, because spectral features are so similar after this age, when compared to younger populations - which actually dominate the spectra of these galaxies. Within this age limitation, the old population could be anything: accumulation of former bursts, or a low intensity, but long lasting continuous star formation episode.

Whatever the star forming history of the old population may be, the important point is that the sum of the early events of star formation turned much more gas into stars than the present one.

\subsection{Age of the young+intermediate population}

The only other free parameter of our study is the age of the young+intermediate population. Figure 14 shows histograms of the best fitting age $e_{y+i}$ of all spectra for which solutions were found (upper panel), and of the oldest young+intermediate population found in each galaxy (lower panel).

The young+intermediate population is a mixture of a certain young bursting population that is able to ionize the interstellar medium (age < $10 \mathrm{Myr}$ ) and - maybe - an intermediate age population (whose age could be anything between $10 \mathrm{Myr}$ and $500 \mathrm{Myr}$ ). 



Fig. 11. Comparison of the indices of the solutions obtained with "BC99" and "Starburst" (crosses). The lines represent identity.

It is noteworthy that a significant fraction of our galaxies $(\simeq 60 \%$ ) present young+intermediate ages greater than $10 \mathrm{Myr}$ (lower panel of Fig. 14), indicating in these cases the very existence of an intermediate age population in addition to the ionizing massive stellar component which necessarily is younger than 10 Myr. Some individual differences exist between the fits with the "Starburst" and "BC99" libraries, especially in the age range from 5 to 50 Myr. However, in general the results are consistent, as could already be seen at the end of the previous section.

As we stated earlier, we do not disentangle possible multiple events in the intermediate age population but only indicate its presence and its average age. Therefore, it does not appear in our modeling by itself, but rather in a weighted mean together with the young population - with weights that are difficult to determine. Despite these drawbacks, some points are, however, worth being stated. First, the mean age distribution of the young+intermediate population, although quite broad, is peaked around 10/20 Myr ("BC99") and 50 Myr ("Starburst"), indicating that the mean age of the intermediate population should be at least of the same order.

To summarise, in $60 \%$ of the galaxies of our sample, we see an intermediate population, whose mean age should be of the order of at least $\simeq 20 / 50$ Myr and more likely $\geq 50$ Myr.

\section{Summary and conclusions}

In this work, we analyse the stellar content of a sample of HII galaxies, of which we have intermediate resolution $(R \sim$ $1000)$ and high enough signal-to-noise spectra with a wavelength range of 3700 to $7500 \AA$, using the continua and certain absorption features of the spectra. To identify stellar 


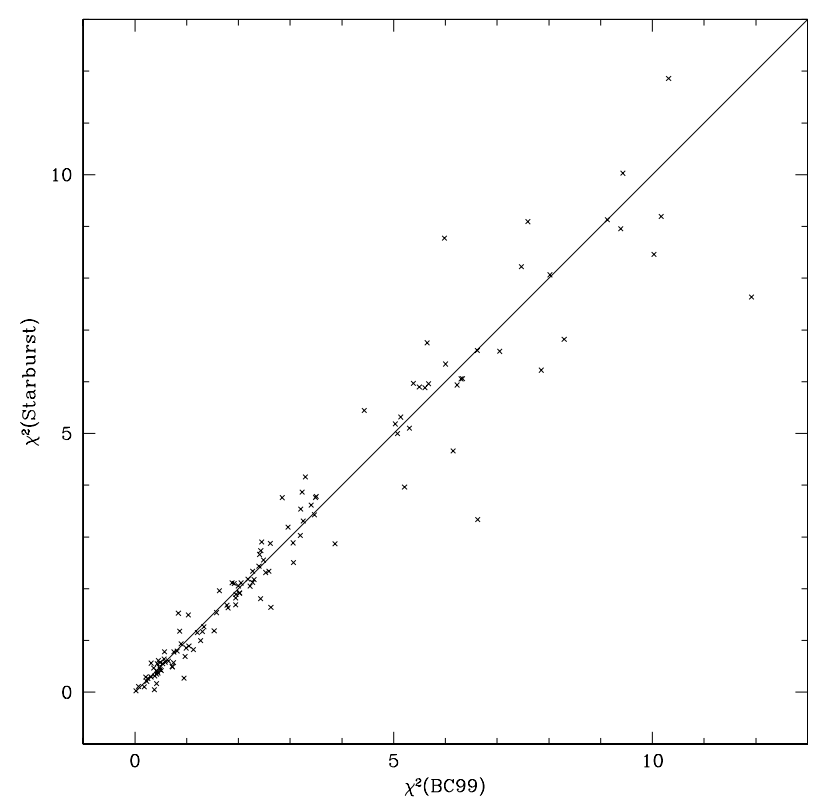

Fig. 12. Comparison of the $\chi^{2}$ of the solutions obtained with "BC99" and "Starburst" (crosses). The line represents identity.

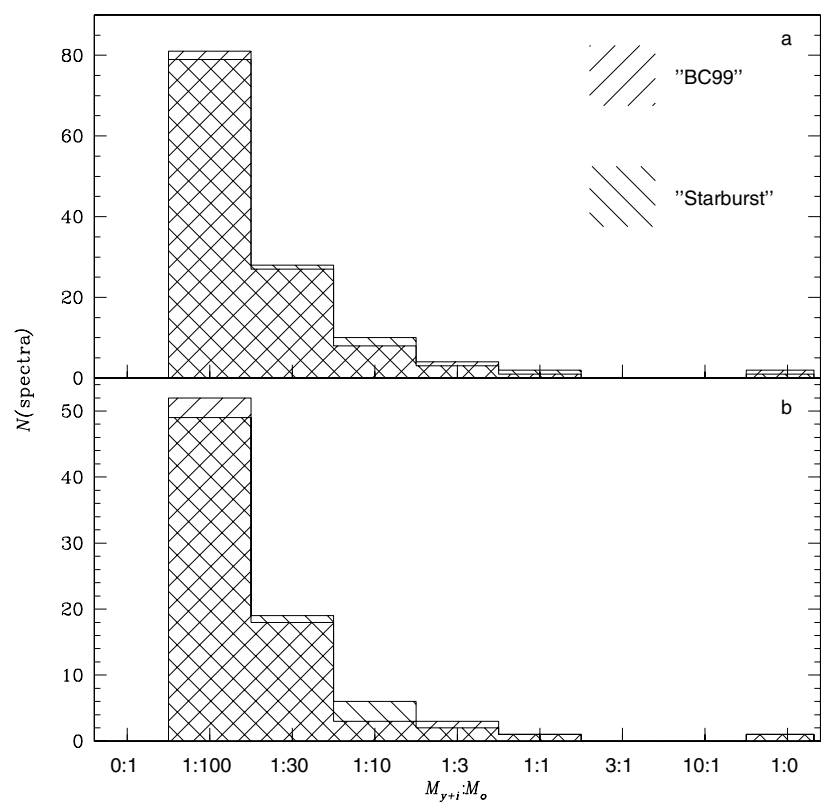

Fig. 13. Histogram of the best fitting $M_{y+i}: M_{\mathrm{o}}$ for the two different libraries. Upper panel: all apertures in all galaxies. Lower panel: one aperture (the lowest $M_{y+i}: M_{\mathrm{o}}$ value) for each galaxy.

populations in these galaxies and to determine their properties (masses, ages, metallicities), we defined six spectral indices (a continuum index and five indices inspired by Lick indices as defined by Worthey et al. (1994); and Worthey (1994), but optimised for the sample spectra). In a best fit procedure, we then determined for each HII galaxy spectrum the combination of two synthetic SSP spectra (one of an old stellar population, and one of a young/intermediate age) that best reproduces the indices of the empirical spectrum.

Although there are certainly more than just two populations in our galaxies, we justify our approach by the fact that we

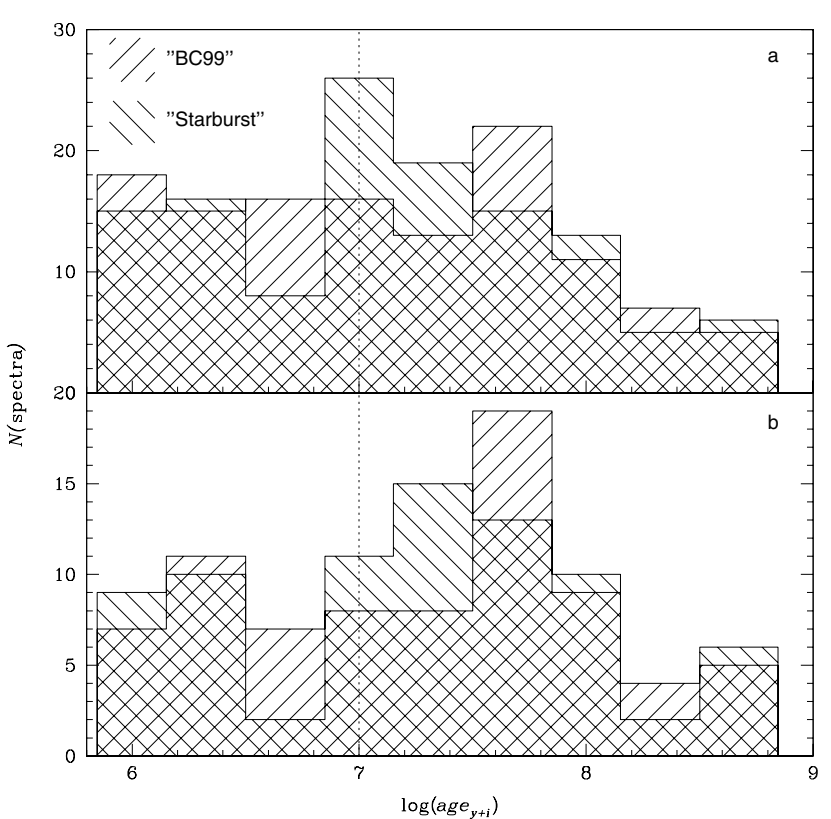

Fig. 14. Histogram of the best fitting $\log \left(\operatorname{age}_{y+i}\right)$ using the two different libraries. Upper panel: all apertures in all galaxies. Lower panel: one aperture (the highest young+intermediate age) for each galaxy. The dotted line corresponds to the maximum duration of an SSP burst, which means that for ages younger than $10^{7} \mathrm{yr}$, the young+intermediate population found could consist of only the young component.

want to use a minimum set of free parameters to describe their observed spectral features.

The main results of the analysis are the following:

(1) In all galaxies of our sample, we detected an old, underlying population $(\geq 1 \mathrm{Gyr})$, generally dominating the stellar mass by a large factor. Although we did not have any extreme metal-poor dwarf galaxies $(\mathrm{O} / \mathrm{H}<7.5)$, which are the most suspected to possess only a young population, some compact HII galaxies were present in our sample, like Fairall 30. Albeit not the least evolved, these galaxies are the ones where the detection of an old population is most difficult, because the young population is also spatially dominant.

(2) In most of our spectra $(\simeq 60 \%)$, we found evidence for an intermediate age population (e.g. $\geq 20 \mathrm{Myr}$ or more likely $\geq 50 \mathrm{Myr}$ ). Because this population cannot be the very young ionizing population (with an age of $\leq 10 \mathrm{Myr}$ ) that we know to be present, this indicates the presence of at least two populations (a young and an intermediate one) connected to the present star forming event.

Our analysis reveals that any realistic modeling of the stellar populations in HII galaxies and subsequent derivation of their physical and evolutionary properties must take into account the presence of at least these three average populations (a young, an intermediate and an old one).

Acknowledgements. This work was supported by the Swiss National Science Foundation. Furthermore, we would like to thank the Fundação Carlos Chagas Filho de Amparo à Pesquisa do Estado do Rio de Janeiro (Faperj) for the help on the infrastructure for this 
project. We also thank the group "Galaxies: Formation, Evolution, and Activity" of the programme PRONEX of the Conselho Nacional de Desenvolvimento Cientifico Tecnológico in Brazil for travel expense allowances. Finally we are grateful to an anonymous referee whose comments greatly helped us to improve the presentation of this paper.

\section{References}

Aloisi, A., Tosi, M., \& Greggio, L. 1999, AJ, 118, 302

Bica, E. 1988, A\&A, 195, 76

Bruzual, A. G., \& Charlot, S. 1993, ApJ, 405, 538

Bruzual, A. G., \& Charlot, S. 2000, Galaxy isochrone spectral synthesis evolution library (private communication)

Bruzual, A. G., \& Charlot, S. 2003, MNRAS, 344, 1000

Buser, R., \& Kurucz, R. L. 1992, A\&A, 264, 557

Calzetti, D., Armus, L., Bohlin, R. C., et al. 2000, ApJ, 533, 682

Charbonnel, C., Meynet, G., Maeder, A., Schaller, G., \& Schaerer, D. 1993, A\&AS, 101, 415

Charlot, S., \& Bruzual, A. G. 1991, ApJ, 367, 126

Cid Fernandes, R., Leão, J. R. S., \& Lacerda, R. R. 2003, MNRAS, 340, 29

Cuisinier, F., Acker, A., \& Köppen, J. 1996, A\&A, 307, 215

Cuisinier, F., Telles, E., \& Kehrig, C. 2004, in preparation

Fluks, M. A., Plez, B., The, P. S., et al. 1994, A\&AS, 105, 311

Gil de Paz, A., Madore, B. F., \& Pevunova, O. 2003, ApJS, 147, 29

Girardi, L., Bressan, A., Bertelli, G., \& Chiosi, C. 2000, A\&AS, 141, 371 ("Padova 2000" isochrones)

Kehrig, C., Telles, E., Cuisinier, F., \& Huziwara, V. 2004, in preparation

Kong, X., Charlot, S., Weiss, A., \& Cheng, F. 2003, A\&A, 403, 877

Legrand, F., Kunth, D., Roy, J.-R., Mas-Hesse, J. M., \& Walsh, J. R. 2000, A\&A, 355, 891

Leitherer, C., Schaerer, D., Goldader, J. D., et al. 1999, ApJS, 123, 3

Lejeune, T., Cuisinier, F., \& Buser, R. 1997, A\&AS, 125, 229

Lejeune, T., Cuisinier, F., \& Buser, R. 1998, A\&AS, 130, 65
Lilly, T., \& Fritze-von Alvensleben, U. 2003, IAU Symp., 221, 80

Meynet, G., Maeder, A., Schaller, G., Schaerer, D., \& Charbonnel, C. 1994, A\&AS, 103, 97

Murakami, L., \& Babul, A. 1999, MNRAS, 309, 161

Östlin, G. 2000, ApJ, 535, L99

Pagel, B. E. J., Simonson, E. A., Terlevich, R. J., \& Edmunds, M. G. 1992, MNRAS, 255, 325

Papaderos, P., Izotov, Y. I., Thuan, T. X., et al. 2002, A\&A, 393, 461

Pickles, A. J. 1998, PASP, 110, 863

Raimann, D., Bica, E., Storchi-Bergmann, T., Melnick, J., \& Schmitt, H. 2000, MNRAS, 314, 295

Salzer, J. J. 1989, ApJ, 347, 152

Sampson, L. 2003, História de Formação Estelar em Galáxias HII, MSc. dissertation, Observatório Nacional, Brazil

Schaerer, D., Charbonnel, C., Meynet, G., Maeder, A., \& Schaller, G. 1993a, A\&AS, 102, 339

Schaerer, D., Meynet, G., Maeder, A., \& Schaller, G. 1993b, A\&AS, 98,523

Schaller, G., Schaerer, D., Meynet, G., \& Maeder, A. 1992, A\&AS, 96, 269

Schmutz, W., Leitherer, C., \& Gruenwald, R. 1992, PASP, 104, 1164

Searle, L., Sargent, W. L. W., \& Bagnuolo, W. G. 1973, AJ, 179, 427

Telles, E. 2002, IAU Symp., 207, 357

Telles, E., \& Terlevich, R. 1995, MNRAS, 275, 1

Telles, E., \& Terlevich, R. 1997, MNRAS, 286, 183

Telles, E., Sampson, L., \& Tapia, M. 2004, in preparation

Tenorio-Tagle, G., Silich, S. A., Kunth, D., Terlevich, E., \& Terlevich, R. 1999, MNRAS, 309, 332

Westera, P. 2001, Ph.D. Thesis, Univ. of Basel, 378

Westera, P., Lejeune, T., Buser, R., Cuisinier, F., \& Bruzual, A. G. 2002, A\&A, 381, 524

Worthey, G. 1994, ApJS, 95, 107

Worthey, G., Faber, S. M., Jesús González, J., \& Burstein, D. 1994, ApJS, 94, 687 


\section{Online Material}




\section{Appendix A: Indices and population parameters of individual spectra}

Table A.1. Indices of individual spectra.

\begin{tabular}{|c|c|c|c|c|c|c|c|}
\hline Galaxy & Type & Cont. i. & $\mathrm{D}(4000)$ & $\mathrm{H}+\mathrm{K}(\mathrm{Ca})$ & $\mathrm{H} \delta$ & Fe4531 & $\mathrm{Mg}_{b}$ \\
\hline Cam08-28A(W) & re & 0.158 & 0.207 & -0.182 & 0.333 & -0.023 & 0.057 \\
\hline Cam08-28A(Cent) & re & -0.648 & 0.054 & 0.072 & 0.120 & -0.027 & 0.093 \\
\hline Cam08-28A(E) & re & -0.691 & 0.050 & 0.079 & 0.043 & 0.034 & 0.116 \\
\hline Cam08-28A & in & -0.751 & 0.052 & 0.066 & 0.054 & 0.007 & 0.103 \\
\hline Cam0357-3915 & in & -0.108 & 0.066 & 0.070 & -0.111 & 0.237 & -0.210 \\
\hline Cam0840+1044 & in & -0.681 & 0.038 & -0.006 & 0.127 & 0.020 & 0.044 \\
\hline Cam0840+1201 & in & -0.569 & -0.029 & 0.001 & 0.015 & -0.016 & 0.119 \\
\hline Cam1543+0907 & in & -0.532 & -0.008 & 0.146 & -0.093 & 0.210 & 0.295 \\
\hline CTS1006 & in & -0.655 & 0.140 & 0.066 & 0.045 & 0.037 & 0.058 \\
\hline CTS1008 & in & -0.564 & -0.099 & -0.004 & -0.139 & -0.014 & 0.048 \\
\hline CTS1011 & in & -0.572 & -0.033 & 0.050 & -0.022 & 0.097 & 0.126 \\
\hline CTS1013 & in & -0.482 & 0.117 & 0.172 & 0.014 & 0.055 & 0.045 \\
\hline CTS1016 & in & -0.602 & 0.039 & 0.151 & 0.053 & 0.038 & 0.030 \\
\hline CTS1017 & in & -0.351 & 0.362 & 0.315 & 0.222 & 0.111 & -0.150 \\
\hline CTS1018 & in & -0.639 & 0.092 & 0.108 & -0.077 & 0.007 & 0.149 \\
\hline CTS1019 & in & -0.551 & -0.024 & 0.082 & -0.050 & 0.014 & 0.076 \\
\hline CTS 1020 & in & -0.516 & 0.682 & 0.055 & 0.369 & -0.008 & -0.025 \\
\hline CTS1022 & in & -0.585 & 0.034 & 0.164 & -0.042 & 0.066 & 0.022 \\
\hline CTS1028 & in & -0.842 & -0.087 & 0.137 & 0.029 & -0.005 & 0.003 \\
\hline CTS1029 & in & -0.461 & -0.045 & 0.142 & 0.076 & 0.029 & 0.059 \\
\hline CTS1033 & in & -0.474 & 0.183 & 0.062 & 0.049 & 0.017 & 0.101 \\
\hline CTS1034 & in & -0.484 & 0.044 & 0.124 & 0.037 & 0.001 & 0.095 \\
\hline CTS1035 & in & -0.392 & -0.119 & -0.196 & 0.132 & 0.038 & -0.029 \\
\hline DDO060(NW) & re & -0.535 & 0.158 & 0.134 & 0.097 & 0.059 & 0.098 \\
\hline DDO060(SE) & re & -0.476 & 0.177 & 0.172 & 0.085 & 0.002 & 0.108 \\
\hline DDO060 & in & -1.338 & 0.031 & 0.202 & -0.039 & -0.183 & 0.149 \\
\hline DDO070(W) & re & -0.534 & 0.110 & 0.185 & 0.031 & 0.000 & -0.156 \\
\hline DDO070(E) & re & -0.558 & 0.080 & 0.137 & 0.006 & 0.011 & -0.068 \\
\hline DDO070 & in & -0.430 & 0.143 & 0.262 & 0.074 & 0.025 & -0.238 \\
\hline DDO075(NE) & re & -0.821 & 0.058 & 0.049 & 0.057 & 0.193 & 0.263 \\
\hline DDO075(CentNE) & re & -0.788 & 0.033 & 0.137 & 0.097 & 0.125 & 0.303 \\
\hline DDO075(CentSW) & re & -0.864 & 0.042 & 0.161 & 0.046 & 0.088 & 0.240 \\
\hline DDO155(NE) & re & -0.726 & 0.126 & 0.175 & -0.018 & 0.026 & -0.015 \\
\hline DDO155(SW) & re & -0.797 & 0.076 & 0.113 & -0.018 & 0.034 & -0.024 \\
\hline DDO155 & in & -1.097 & -0.044 & 0.016 & 0.059 & 0.039 & -0.056 \\
\hline ESO289IG037 & in & -0.482 & 0.188 & 0.132 & 0.056 & 0.051 & 0.047 \\
\hline ESO533G014 & in & -0.503 & 0.152 & 0.184 & 0.082 & 0.049 & 0.073 \\
\hline Fairall30 & in & -0.585 & 0.091 & 0.110 & 0.010 & 0.031 & 0.078 \\
\hline Haro24 & in & -0.721 & 0.060 & 0.062 & 0.053 & 0.031 & 0.053 \\
\hline Haro25 & in & -0.524 & 0.123 & 0.108 & 0.036 & 0.032 & 0.063 \\
\hline Haro30 & in & -0.345 & 0.124 & 0.155 & 0.060 & 0.030 & 0.095 \\
\hline IC5154(N) & re & -0.627 & 0.148 & -0.028 & -0.003 & 0.049 & 0.127 \\
\hline IC5154(S) & re & -0.453 & 0.193 & 0.140 & 0.069 & 0.058 & 0.057 \\
\hline IC5154 & in & -0.491 & 0.174 & 0.096 & 0.064 & 0.057 & 0.082 \\
\hline Marseille01 & in & -0.524 & 0.151 & 0.038 & 0.027 & 0.043 & 0.130 \\
\hline Marseille68 & in & -0.392 & 0.126 & 0.118 & 0.011 & 0.043 & 0.104 \\
\hline Marseille88 & in & -0.576 & 0.012 & 0.002 & 0.091 & 0.012 & 0.041 \\
\hline MBG00463-0239 & in & -0.420 & 0.181 & 0.212 & 0.018 & 0.036 & 0.089 \\
\hline MBG02411-1457 & in & -0.369 & 0.318 & 0.258 & 0.102 & 0.061 & 0.093 \\
\hline MBG20533-4410 & in & -0.282 & 0.034 & -0.036 & -0.034 & 0.022 & -0.027 \\
\hline
\end{tabular}


Table A.1. continued.

\begin{tabular}{|c|c|c|c|c|c|c|c|}
\hline Galaxy & Type & Cont. i. & $\mathrm{D}(4000)$ & $\mathrm{H}+\mathrm{K}(\mathrm{Ca})$ & $\mathrm{H} \delta$ & $\mathrm{Fe} 4531$ & $\mathrm{Mg}_{b}$ \\
\hline MBG21567-1645(E) & re & -0.527 & 0.245 & 0.195 & 0.034 & 0.088 & 0.129 \\
\hline MBG21567-1645(Cent) & re & -0.606 & 0.309 & 0.287 & 0.010 & 0.069 & 0.125 \\
\hline MBG21567-1645(W) & re & -0.580 & 0.262 & 0.251 & 0.030 & 0.071 & 0.130 \\
\hline MBG21567-1645 & in & -0.504 & 0.155 & 0.179 & 0.044 & 0.077 & 0.103 \\
\hline MBG22012-1550(E) & re & -0.796 & 0.079 & 0.132 & 0.096 & 0.032 & 0.064 \\
\hline MBG22012-1550(W) & re & -0.742 & 0.127 & 0.153 & 0.042 & 0.074 & 0.041 \\
\hline MBG22012-1550 & in & -0.589 & 0.126 & 0.148 & 0.055 & 0.059 & 0.039 \\
\hline MBG23121-3807 & in & -0.490 & 0.209 & 0.181 & 0.052 & 0.050 & 0.080 \\
\hline MCG0157017 & in & -0.474 & 0.035 & 0.124 & 0.053 & 0.049 & 0.073 \\
\hline Mrk36 & in & -0.699 & -0.004 & 0.032 & 0.027 & 0.009 & -0.016 \\
\hline Mrk710(SW) & re & -1.183 & -0.300 & 0.224 & 0.073 & -0.026 & -0.442 \\
\hline Mrk710(CentSW) & re & -0.170 & 0.278 & 0.193 & 0.112 & -0.172 & 0.167 \\
\hline Mrk710(CentNE) & re & -0.594 & 0.054 & 0.038 & 0.049 & 0.007 & 0.111 \\
\hline Mrk710(NE) & re & -0.622 & 0.036 & 0.042 & 0.009 & 0.028 & 0.061 \\
\hline Mrk710 & in & -0.611 & 0.035 & 0.053 & 0.021 & 0.036 & 0.051 \\
\hline Mrk711 & in & -0.309 & 0.147 & 0.274 & -0.042 & 0.041 & 0.104 \\
\hline Mrk1201 & in & -0.250 & 0.212 & 0.308 & -0.151 & -0.012 & 0.167 \\
\hline Mrk1318 & in & -0.503 & 0.117 & 0.127 & 0.047 & 0.027 & 0.081 \\
\hline NGC7323(E) & re & -0.465 & 0.111 & 0.049 & 0.069 & 0.013 & 0.259 \\
\hline NGC7323(W) & re & -0.435 & 0.132 & 0.036 & 0.024 & 0.026 & 0.209 \\
\hline NGC7323 & in & -0.641 & 0.129 & -0.001 & 0.018 & 0.071 & 0.032 \\
\hline POX186 & in & -0.424 & 0.087 & -0.251 & 0.037 & -0.054 & 0.250 \\
\hline Tol0104-388(NW) & re & -0.563 & -0.109 & -0.093 & 0.044 & 0.057 & 0.090 \\
\hline Tol0104-388(SE) & re & -0.952 & 0.104 & -0.017 & 0.040 & 0.048 & 0.065 \\
\hline Tol0117-414EW & re & -0.575 & 0.037 & 0.160 & 0.086 & 0.022 & 0.115 \\
\hline Tol0117-414NS(N) & re & -0.731 & 0.051 & 0.111 & -0.001 & 0.009 & 0.120 \\
\hline Tol0117-414NS(CentN) & re & -0.585 & 0.101 & 0.194 & 0.056 & 0.047 & 0.063 \\
\hline Tol0117-414NS(CentS) & re & -0.484 & 0.065 & 0.021 & 0.037 & -0.002 & 0.132 \\
\hline Tol0117-414NS(S) & re & -0.772 & 0.018 & 0.273 & 0.060 & 0.118 & -0.004 \\
\hline Tol0140-420 & in & -0.474 & 0.002 & 0.059 & 0.060 & 0.076 & 0.122 \\
\hline Tol0226-390 & in & -0.630 & 0.011 & -0.021 & 0.009 & 0.013 & 0.114 \\
\hline Tol0306-405 & in & -0.654 & 0.031 & 0.019 & 0.010 & 0.048 & 0.063 \\
\hline Tol0341-407(E) & re & -0.559 & 0.000 & 0.095 & 0.076 & 0.116 & 0.054 \\
\hline Tol0341-407(W) & re & -0.475 & -0.032 & 0.059 & 0.099 & 0.086 & 0.057 \\
\hline Tol0341-407 & in & -0.179 & -0.101 & -0.044 & 0.124 & 0.070 & 0.080 \\
\hline Tol0440-381 & in & -0.582 & -0.027 & 0.078 & 0.118 & 0.029 & 0.089 \\
\hline Tol0505-387 & in & -0.086 & 0.036 & 0.037 & 0.135 & 0.038 & 0.043 \\
\hline Tol0510-400 & in & -0.352 & -0.082 & -0.049 & 0.047 & 0.071 & 0.185 \\
\hline Tol0528-383(W) & re & -0.771 & 0.118 & -0.118 & 0.051 & 0.002 & 0.090 \\
\hline Tol0528-383(E) & re & -0.575 & 0.158 & -0.022 & 0.032 & -0.021 & 0.056 \\
\hline Tol0528-383 & in & -0.476 & 0.156 & 0.085 & 0.084 & -0.001 & 0.009 \\
\hline Tol0538-416 & in & -0.359 & -0.081 & -0.019 & 0.004 & 0.102 & 0.033 \\
\hline Tol0610-387 & in & -0.698 & -0.038 & 0.132 & 0.158 & -0.040 & -0.019 \\
\hline Tol0633-415 & in & -0.582 & -0.043 & 0.511 & 0.020 & 0.322 & 0.145 \\
\hline Tol0645-376 & in & -0.474 & 0.073 & 0.092 & 0.068 & 0.042 & 0.028 \\
\hline Tol0957-278(NW) & re & -0.582 & 0.010 & 0.024 & 0.012 & 0.029 & 0.048 \\
\hline Tol0957-278(SE) & re & -0.631 & 0.015 & 0.035 & 0.031 & 0.010 & 0.033 \\
\hline Tol0957-278 & in & -0.651 & 0.019 & 0.038 & 0.038 & 0.005 & 0.035 \\
\hline Tol1004-296(NW) & re & -0.538 & 0.067 & 0.057 & 0.030 & 0.018 & 0.052 \\
\hline Tol1004-296(SE) & re & -0.594 & 0.046 & 0.045 & 0.022 & 0.019 & 0.046 \\
\hline Tol1004-296 & in & -1.895 & -0.060 & 0.032 & 0.004 & 0.027 & 0.036 \\
\hline
\end{tabular}


Table A.1. continued.

\begin{tabular}{|c|c|c|c|c|c|c|c|}
\hline Galaxy & Type & Cont. i. & $\mathrm{D}(4000)$ & $\mathrm{H}+\mathrm{K}(\mathrm{Ca})$ & $\mathrm{H} \delta$ & $\mathrm{Fe} 4531$ & $\mathrm{Mg}_{b}$ \\
\hline Tol1008-286(SE) & re & -0.261 & 0.449 & -0.062 & -0.227 & 0.026 & -0.189 \\
\hline Tol1008-286(NW) & re & -0.974 & -0.037 & 0.046 & -0.048 & -0.122 & 0.439 \\
\hline Tol1008-286 & in & -0.369 & 0.150 & 0.149 & -0.670 & 0.178 & 0.071 \\
\hline Tol1025-285 & in & -0.589 & 0.083 & 0.168 & 0.002 & -0.021 & 0.148 \\
\hline Tol1146-333 & in & -0.419 & 0.178 & 0.147 & 0.073 & 0.100 & -0.014 \\
\hline Tol1147-283 & in & -0.460 & 0.062 & 0.161 & 0.047 & 0.098 & 0.127 \\
\hline Tol1223-359 & in & -0.723 & -0.105 & -0.194 & 0.000 & 0.026 & -0.372 \\
\hline Tol1345-420 & in & -0.430 & 0.119 & 0.080 & 0.065 & 0.038 & 0.073 \\
\hline Tol1455-284(E) & re & -0.121 & -0.036 & -0.012 & 0.082 & -0.054 & -0.041 \\
\hline Tol1455-284(W) & re & 0.126 & 0.152 & 0.023 & 0.072 & -0.006 & 0.076 \\
\hline Tol1455-284 & in & 0.102 & 0.130 & 0.144 & -0.013 & 0.012 & 0.094 \\
\hline Tol1457-262E(NW) & re & -0.484 & 0.026 & 0.060 & 0.007 & 0.024 & 0.079 \\
\hline Tol1457-262E(SE) & re & -0.503 & 0.021 & 0.063 & 0.007 & 0.029 & 0.074 \\
\hline Tol1457-262E & in & -0.536 & 0.011 & 0.034 & -0.005 & 0.052 & 0.076 \\
\hline Tol1457-262W(W) & re & -0.553 & 0.001 & 0.086 & 0.048 & -0.015 & 0.021 \\
\hline Tol1457-262W(CentW) & re & -0.571 & 0.016 & 0.096 & 0.049 & 0.008 & 0.051 \\
\hline Tol1457-262W(Cent) & re & -0.472 & 0.082 & 0.119 & 0.071 & 0.061 & 0.056 \\
\hline Tol1457-262W(CentE) & re & -0.513 & 0.055 & 0.103 & 0.063 & 0.026 & 0.050 \\
\hline Tol1457-262W(E) & re & -0.558 & 0.030 & 0.096 & 0.097 & -0.041 & 0.011 \\
\hline Tol1457-262W & in & -0.145 & 0.315 & -0.062 & -0.013 & -0.009 & 0.037 \\
\hline Tol1924-416(W) & re & -0.656 & -0.018 & 0.043 & -0.010 & 0.027 & 0.045 \\
\hline Tol1924-416(E) & re & -0.761 & -0.013 & 0.068 & -0.018 & 0.026 & 0.051 \\
\hline Tol1924-416 & in & -0.679 & -0.016 & 0.054 & -0.014 & 0.027 & 0.048 \\
\hline Tol1937-423 & in & -0.596 & 0.092 & 0.209 & 0.075 & 0.039 & 0.047 \\
\hline Tol2019-405 & in & -0.382 & 0.128 & 0.045 & 0.025 & 0.042 & 0.017 \\
\hline Tol2122-408 & in & -0.410 & 0.183 & -0.017 & 0.062 & 0.029 & 0.057 \\
\hline Tol2138-397 & in & -0.554 & 0.177 & 0.221 & -0.038 & 0.090 & 0.097 \\
\hline Tol2146-391 & in & -0.725 & 0.258 & 0.045 & -0.090 & -0.270 & -0.215 \\
\hline Tol2240-384 & in & -0.360 & -0.059 & -0.141 & 0.035 & -0.100 & -0.149 \\
\hline UM69(E) & re & -1.314 & 0.020 & 0.112 & 0.056 & 0.044 & 0.029 \\
\hline UM69(Cent) & re & -0.650 & 0.077 & 0.122 & 0.066 & 0.047 & 0.047 \\
\hline UM69(W) & re & -0.744 & -0.026 & 0.086 & 0.052 & 0.051 & 0.050 \\
\hline UM69 & in & -1.058 & 0.013 & 0.108 & 0.058 & 0.047 & 0.041 \\
\hline UM137(E) & re & -1.467 & 0.086 & 0.036 & 0.150 & 0.069 & 0.044 \\
\hline UM137(CentE) & re & -0.619 & 0.148 & 0.104 & 0.107 & 0.033 & 0.047 \\
\hline UM137(CentW) & re & -0.587 & 0.123 & 0.085 & 0.116 & 0.035 & 0.039 \\
\hline UM137(W) & re & -0.423 & 0.041 & 0.060 & 0.137 & 0.053 & 0.020 \\
\hline UM137 & in & -0.462 & 0.042 & 0.071 & 0.142 & 0.016 & 0.045 \\
\hline UM151 & in & -0.482 & 0.084 & 0.547 & -0.084 & 0.073 & 0.067 \\
\hline UM160(E) & re & -0.709 & 0.019 & 0.106 & 0.014 & 0.141 & 0.238 \\
\hline UM160(Cent) & re & -0.865 & -0.051 & 0.071 & 0.039 & 0.066 & 0.165 \\
\hline UM160(W) & re & -0.633 & -0.012 & 0.131 & 0.059 & 0.016 & 0.104 \\
\hline UM160 & in & -0.690 & -0.032 & 0.114 & 0.019 & 0.038 & 0.171 \\
\hline UM166 & in & -0.460 & 0.242 & 0.182 & 0.041 & 0.062 & 0.146 \\
\hline UM191 & in & -0.593 & 0.102 & 0.132 & 0.057 & 0.039 & 0.073 \\
\hline UM238(E) & re & -0.584 & 0.154 & 0.149 & 0.030 & 0.024 & 0.090 \\
\hline UM238(W) & re & -0.177 & 0.147 & 0.103 & -0.005 & 0.121 & 0.021 \\
\hline UM238 & in & -0.518 & 0.139 & 0.139 & 0.032 & 0.057 & 0.060 \\
\hline UM306 & in & -0.520 & 0.233 & 0.145 & 0.067 & 0.026 & 0.041 \\
\hline UM307 & in & -0.709 & 0.071 & 0.125 & 0.059 & 0.057 & 0.085 \\
\hline
\end{tabular}


P. Westera et al.: Stellar populations in HII galaxies, Online Material p 5

Table A.1. continued.

\begin{tabular}{|c|c|c|c|c|c|c|c|}
\hline Galaxy & Type & Cont. i. & $\overline{\mathrm{D}(4000)}$ & $\mathrm{H}+\mathrm{K}(\mathrm{Ca})$ & $\overline{\mathrm{H} \delta}$ & Fe4531 & $\overline{\mathrm{Mg}_{b}}$ \\
\hline UM323 & in & -1.140 & 0.020 & 0.046 & 0.085 & 0.040 & 0.038 \\
\hline UM382 & in & -0.512 & 0.014 & 0.123 & 0.207 & 0.037 & -0.191 \\
\hline UM391 & in & -0.158 & 0.094 & -0.241 & -0.030 & -0.029 & 0.001 \\
\hline UM395 & in & -0.651 & 0.125 & 0.132 & 0.100 & 0.059 & 0.081 \\
\hline UM396 & in & -0.665 & 0.017 & 0.000 & -0.001 & 0.038 & -0.082 \\
\hline UM408 & in & -0.632 & 0.105 & -0.152 & -0.002 & 0.046 & 0.042 \\
\hline UM417 & in & -0.550 & 0.126 & 0.438 & -0.024 & 0.175 & -0.173 \\
\hline UM439(SE) & re & -0.410 & 0.100 & 0.136 & -0.044 & 0.045 & 0.024 \\
\hline UM439(Cent) & re & -0.585 & 0.052 & 0.103 & 0.073 & 0.040 & 0.046 \\
\hline UM439(NW) & re & -0.579 & 0.053 & 0.106 & 0.043 & 0.051 & 0.021 \\
\hline UM439 & in & -0.634 & 0.036 & 0.093 & 0.019 & 0.048 & 0.003 \\
\hline UM448 & in & -0.470 & 0.104 & 0.125 & 0.034 & 0.036 & 0.098 \\
\hline UM448(extension NE-SW) & in & -0.504 & 0.065 & 0.094 & 0.012 & 0.040 & 0.119 \\
\hline UM455(NW) & re & -0.469 & 0.102 & 0.067 & -0.020 & 0.026 & -0.018 \\
\hline UM455(SE) & re & -0.116 & 0.049 & 0.168 & -0.071 & 0.098 & -0.029 \\
\hline UM455 & in & 0.465 & 0.005 & 0.417 & -0.034 & 0.060 & -0.003 \\
\hline UM456(NE) & re & -0.742 & -0.011 & 0.108 & 0.021 & 0.101 & 0.041 \\
\hline UM456(Cent) & re & -0.754 & -0.012 & 0.068 & 0.027 & 0.069 & 0.036 \\
\hline UM456(SW) & re & -0.750 & -0.034 & 0.048 & 0.029 & 0.024 & -0.002 \\
\hline UM456 & in & -0.732 & 0.021 & 0.080 & 0.026 & 0.047 & 0.064 \\
\hline UM461(E) & re & -0.686 & 0.061 & -0.001 & -0.091 & -0.087 & 0.047 \\
\hline UM461(W) & re & -0.728 & 0.043 & 0.053 & -0.084 & -0.100 & 0.034 \\
\hline UM461 & in & -0.808 & -0.007 & 0.182 & -0.066 & -0.036 & 0.001 \\
\hline UM462(SW) & re & -0.884 & -0.048 & 0.002 & 0.011 & 0.040 & 0.028 \\
\hline UM462(NE) & re & -0.755 & -0.017 & 0.019 & 0.014 & 0.031 & 0.085 \\
\hline UM462 & in & -0.796 & -0.024 & 0.004 & 0.014 & 0.036 & 0.058 \\
\hline UM463 & in & -0.669 & -0.764 & -0.243 & -0.058 & 0.069 & 0.257 \\
\hline UM477 & in & -0.229 & 0.202 & 0.160 & 0.017 & 0.046 & 0.107 \\
\hline UM483 & in & -0.482 & 0.078 & 0.020 & 0.078 & 0.000 & 0.123 \\
\hline UM499(W) & re & -0.569 & 0.080 & 0.102 & 0.088 & 0.039 & 0.095 \\
\hline UM499(E) & re & -0.447 & 0.155 & 0.131 & 0.019 & 0.045 & 0.083 \\
\hline UM499 & in & -0.437 & 0.155 & 0.136 & 0.023 & 0.046 & 0.087 \\
\hline UM533(E) & re & -0.655 & 0.100 & 0.097 & 0.019 & 0.042 & 0.108 \\
\hline UM533(W) & re & -0.535 & 0.004 & -0.016 & -0.019 & 0.055 & 0.034 \\
\hline UM533 & in & -0.482 & 0.058 & 0.006 & -0.025 & 0.046 & 0.044 \\
\hline UM559(giant E-RHII) & re & -0.784 & 0.073 & -0.260 & -0.088 & -0.115 & 0.392 \\
\hline UM559(E) & re & -0.638 & -0.079 & -0.586 & 0.210 & -0.975 & 0.959 \\
\hline UM559(Cent) & re & -0.683 & 0.158 & 0.240 & 0.047 & 0.087 & 0.118 \\
\hline UM559 & in & -0.459 & 0.154 & 0.139 & -0.211 & 0.307 & 0.381 \\
\hline UM570 & in & -0.674 & 0.464 & 1.274 & -0.274 & 0.732 & -1.148 \\
\hline UM598E(SW) & re & -0.217 & 0.147 & 0.079 & 0.117 & 0.014 & 0.015 \\
\hline UM598E(Cent) & re & -0.485 & 0.291 & 0.274 & 0.013 & 0.057 & 0.072 \\
\hline UM598E(NE) & re & -0.935 & 0.169 & 0.184 & 0.050 & 0.047 & 0.054 \\
\hline UM598E & in & -0.858 & 0.106 & 0.172 & 0.056 & 0.054 & 0.043 \\
\hline UM598W(SW) & re & -0.578 & 0.080 & 0.069 & 0.007 & 0.051 & 0.084 \\
\hline UM598W(CentSW) & re & -0.339 & 0.145 & 0.163 & 0.061 & 0.041 & 0.101 \\
\hline UM598W(CentNE) & re & -0.392 & 0.264 & 0.197 & 0.015 & 0.060 & 0.130 \\
\hline UM598W(NE) & re & -0.473 & 0.140 & 0.130 & 0.040 & 0.042 & 0.086 \\
\hline UM598W & in & -0.574 & 0.103 & 0.119 & 0.042 & 0.018 & 0.049 \\
\hline IIZw40 & in & -0.363 & -0.024 & 0.017 & -0.055 & 0.020 & 0.059 \\
\hline
\end{tabular}


P. Westera et al.: Stellar populations in HII galaxies, Online Material p 6

Table A.2. Population parameters of individual spectra.

\begin{tabular}{|c|c|c|c|c|c|}
\hline \multirow[t]{2}{*}{ Galaxy } & \multirow[t]{2}{*}[\mathrm{Fe}/\mathrm{H}]{$_{y+i}$} & \multicolumn{2}{|c|}{ “ВC99" } & \multicolumn{2}{|c|}{ "Starburst" } \\
\hline & & $M_{y+i}: M_{\mathrm{o}}$ & $\operatorname{age}_{y+i}[\mathrm{Myr}]$ & $M_{y+i}: M_{\mathrm{o}}$ & $\operatorname{age}_{y+i}[\mathrm{Myr}]$ \\
\hline Cam08-28A(Cent) & -1 & $1: 10$ & 102 & $1: 10$ & 100 \\
\hline Cam08-28A(E) & -1 & $1: 100$ & 10 & $1: 100$ & 10 \\
\hline Cam08-28A & -0.744 & $1: 100$ & 10 & $1: 100$ & 10 \\
\hline Cam0840+1044 & -1.140 & $1: 3$ & 102 & $1: 3$ & 100 \\
\hline Cam0840+1201 & -0.894 & $1: 30$ & 10 & $1: 100$ & 2 \\
\hline Cam1543+0907 & -1.163 & $1: 100$ & 2 & $1: 100$ & 1 \\
\hline CTS1008 & -0.713 & $1: 30$ & 1 & $1: 30$ & 1 \\
\hline CTS1011 & -0.692 & $1: 100$ & 2 & $1: 100$ & 2 \\
\hline CTS1016 & -1 & $1: 100$ & 10 & $1: 100$ & 20 \\
\hline CTS1018 & -0.900 & $1: 100$ & 10 & $1: 100$ & 20 \\
\hline CTS1019 & -0.646 & $1: 100$ & 2 & $1: 100$ & 1 \\
\hline CTS1022 & -0.781 & $1: 100$ & 1 & $1: 100$ & 10 \\
\hline CTS1028 & -0.820 & $1: 30$ & 2 & $1: 30$ & 2 \\
\hline CTS1029 & -1 & $1: 100$ & 2 & $1: 100$ & 10 \\
\hline CTS1033 & -0.862 & $1: 100$ & 102 & $1: 30$ & 200 \\
\hline CTS1034 & -0.907 & $1: 100$ & 10 & $1: 100$ & 20 \\
\hline DDO060(NW) & -1 & $1: 30$ & 203 & $1: 30$ & 200 \\
\hline DDO060(SE) & -1 & $1: 10$ & 509 & $1: 10$ & 500 \\
\hline DDO070(E) & -1.410 & $1: 100$ & 10 & $1: 100$ & 20 \\
\hline DDO075(CentSW) & -1 & $1: 100$ & 5 & $1: 100$ & 5 \\
\hline DDO155(SW) & -1.229 & $1: 100$ & 5 & $1: 100$ & 10 \\
\hline DDO155 & -1.109 & $1: 0$ & 20 & $1: 1$ & 5 \\
\hline ESO289IG037 & -1 & $1: 100$ & 203 & $1: 100$ & 100 \\
\hline Fairall30 & -1 & $1: 100$ & 20 & $1: 100$ & 20 \\
\hline Haro24 & -1 & $1: 30$ & 50 & $1: 100$ & 20 \\
\hline Haro25 & -1 & $1: 100$ & 50 & $1: 100$ & 50 \\
\hline Haro30 & -1.201 & $1: 100$ & 50 & $1: 100$ & 50 \\
\hline IC5154(N) & -1 & $1: 100$ & 50 & $1: 30$ & 100 \\
\hline Marseille68 & -1 & $1: 100$ & 50 & $1: 100$ & 50 \\
\hline Marseille88 & -0.851 & $1: 3$ & 50 & $1: 3$ & 50 \\
\hline MBG20533-4410 & -1 & $1: 100$ & 1 & $1: 100$ & 1 \\
\hline MBG21567-1645 & -1 & $1: 100$ & 102 & $1: 100$ & 100 \\
\hline MBG22012-1550(E) & -1 & $1: 30$ & 50 & $1: 30$ & 50 \\
\hline MCG0157017 & -1 & $1: 100$ & 10 & $1: 100$ & 20 \\
\hline Mrk36 & -0.976 & $1: 100$ & 5 & $1: 100$ & 2 \\
\hline Mrk710(CentNE) & -1 & $1: 10$ & 50 & $1: 100$ & 10 \\
\hline Mrk710(NE) & -1 & $1: 30$ & 20 & $1: 100$ & 2 \\
\hline Mrk710 & -1 & $1: 100$ & 10 & $1: 100$ & 10 \\
\hline Mrk1318 & -0.598 & $1: 100$ & 50 & $1: 30$ & 50 \\
\hline NGC7323(E) & -1 & $1: 30$ & 102 & $1: 30$ & 100 \\
\hline NGC7323(W) & -1 & $1: 100$ & 50 & $1: 30$ & 100 \\
\hline
\end{tabular}


Table A.2. continued.

\begin{tabular}{|c|c|c|c|c|c|}
\hline \multirow[t]{2}{*}{ Galaxy } & \multirow[t]{2}{*}[\mathrm{Fe}/\mathrm{H}]{$_{y+i}$} & \multicolumn{2}{|c|}{ "BC99" } & \multicolumn{2}{|c|}{ "Starburst" } \\
\hline & & $M_{y+i}: M_{\mathrm{o}}$ & $\operatorname{age}_{y+i}[\mathrm{Myr}]$ & $M_{y+i}: M_{\mathrm{o}}$ & $\operatorname{age}_{y+i}[\mathrm{Myr}]$ \\
\hline Tol0104-388(NW) & -0.908 & $1: 30$ & 2 & $1: 10$ & 1 \\
\hline Tol0117-414EW & -1 & $1: 30$ & 50 & $1: 100$ & 20 \\
\hline Tol0117-414NS(N) & -1 & $1: 100$ & 1 & $1: 100$ & 10 \\
\hline Tol0117-414NS(CentS) & -1 & $1: 100$ & 10 & $1: 100$ & 20 \\
\hline Tol0140-420 & -0.812 & $1: 100$ & 1 & $1: 100$ & 10 \\
\hline Tol0226-390 & -0.725 & $1: 100$ & 2 & $1: 100$ & 1 \\
\hline Tol0306-405 & -0.728 & $1: 100$ & 1 & $1: 100$ & 10 \\
\hline Tol0341-407(E) & -0.738 & $1: 30$ & 20 & $1: 100$ & 10 \\
\hline Tol0341-407(W) & -0.755 & $1: 30$ & 20 & $1: 100$ & 1 \\
\hline Tol0440-381 & -0.906 & $1: 3$ & 50 & $1: 30$ & 20 \\
\hline Tol0510-400 & -1 & $1: 30$ & 10 & $1: 30$ & 1 \\
\hline Tol0528-383(W) & -0.863 & $1: 3$ & 203 & $1: 3$ & 200 \\
\hline Tol0528-383(E) & -0.722 & $1: 30$ & 102 & $1: 30$ & 100 \\
\hline Tol0528-383 & -0.912 & $1: 30$ & 203 & $1: 30$ & 200 \\
\hline Tol0538-416 & -0.870 & $1: 100$ & 2 & $1: 100$ & 2 \\
\hline Tol0610-387 & -1 & $1: 1$ & 50 & $1: 1$ & 50 \\
\hline Tol0645-376 & -1.100 & $1: 100$ & 20 & $1: 100$ & 20 \\
\hline Tol0957-278(NW) & -0.761 & $1: 100$ & 1 & $1: 100$ & 1 \\
\hline Tol0957-278(SE) & -0.972 & $1: 100$ & 1 & $1: 100$ & 10 \\
\hline Tol0957-278 & -0.846 & $1: 100$ & 1 & $1: 100$ & 10 \\
\hline Tol1004-296(NW) & -0.592 & $1: 100$ & 20 & $1: 100$ & 20 \\
\hline Tol1004-296(SE) & -0.666 & $1: 100$ & 10 & $1: 100$ & 10 \\
\hline Tol1008-286(NW) & -1 & $1: 30$ & 1 & $1: 10$ & 2 \\
\hline Tol1147-283 & -0.992 & $1: 100$ & 20 & $1: 100$ & 20 \\
\hline Tol1223-359 & -1 & $1: 30$ & 2 & $1: 0$ & 1 \\
\hline Tol1345-420 & -0.610 & $1: 30$ & 102 & $1: 30$ & 50 \\
\hline Tol1457-262E(NW) & -0.653 & $1: 100$ & 10 & $1: 100$ & 10 \\
\hline Tol1457-262E(SE) & -0.805 & $1: 100$ & 1 & $1: 100$ & 10 \\
\hline Tol1457-262E & -0.705 & $1: 100$ & 1 & $1: 100$ & 1 \\
\hline Tol1457-262W(W) & -1 & $1: 100$ & 1 & $1: 100$ & 10 \\
\hline Tol1457-262W(CentW) & -0.903 & $1: 100$ & 1 & $1: 100$ & 10 \\
\hline Tol1457-262W(Cent) & -0.737 & $1: 100$ & 20 & $1: 30$ & 50 \\
\hline Tol1457-262W(CentE) & -0.787 & $1: 30$ & 50 & $1: 100$ & 20 \\
\hline Tol1457-262W & -0.748 & $1: 100$ & 509 & $1: 100$ & 500 \\
\hline Tol1924-416(W) & -0.855 & $1: 100$ & 2 & $1: 100$ & 2 \\
\hline Tol1924-416(E) & -0.874 & $1: 100$ & 5 & $1: 100$ & 2 \\
\hline Tol1924-416 & -0.863 & $1: 100$ & 2 & $1: 100$ & 2 \\
\hline Tol1937-423 & -1 & $1: 30$ & 50 & $1: 30$ & 50 \\
\hline Tol2019-405 & -0.882 & $1: 100$ & 50 & $1: 30$ & 100 \\
\hline Tol2122-408 & -1 & $1: 10$ & 509 & $1: 10$ & 500 \\
\hline
\end{tabular}


P. Westera et al.: Stellar populations in HII galaxies, Online Material p 8

Table A.2. continued.

\begin{tabular}{|c|c|c|c|c|c|}
\hline \multirow[t]{2}{*}{$\overline{\text { Galaxy }}$} & \multirow[t]{2}{*}[\mathrm{Fe}/\mathrm{H}]{$_{y+i}$} & \multicolumn{2}{|c|}{ "BC99" } & \multicolumn{2}{|c|}{ "Starburst" } \\
\hline & & $M_{y+i}: M_{\mathrm{o}}$ & $\operatorname{age}_{y+i}[\mathrm{Myr}]$ & $M_{y+i}: M_{\mathrm{o}}$ & $\operatorname{age}_{y+i}[\mathrm{Myr}]$ \\
\hline UM69(E) & -0.818 & $1: 100$ & 5 & $1: 30$ & 5 \\
\hline UM69(Cent) & -1.147 & $1: 100$ & 20 & $1: 100$ & 20 \\
\hline UM69(W) & -1 & $1: 100$ & 5 & $1: 100$ & 5 \\
\hline UM69 & -0.672 & $1: 100$ & 5 & $1: 30$ & 5 \\
\hline UM137(CentE) & -1 & $1: 30$ & 203 & $1: 10$ & 500 \\
\hline UM137(CentW) & -1 & $1: 10$ & 203 & $1: 10$ & 200 \\
\hline UM137(W) & -1 & $1: 10$ & 102 & $1: 10$ & 100 \\
\hline UM160(E) & -1 & $1: 100$ & 5 & $1: 100$ & 10 \\
\hline UM160(Cent) & -1 & $1: 30$ & 1 & $1: 30$ & 10 \\
\hline UM160(W) & -1 & $1: 100$ & 2 & $1: 100$ & 10 \\
\hline UM160 & -1 & $1: 100$ & 5 & $1: 100$ & 2 \\
\hline UM191 & -1 & $1: 100$ & 50 & $1: 30$ & 100 \\
\hline UM323 & -0.950 & $1: 0$ & 50 & $1: 30$ & 5 \\
\hline UM395 & -1 & $1: 30$ & 203 & $1: 30$ & 100 \\
\hline UM396 & -0.688 & $1: 100$ & 2 & $1: 100$ & 1 \\
\hline UM408 & -0.846 & $1: 10$ & 102 & $1: 10$ & 100 \\
\hline UM439(Cent) & -1.076 & $1: 30$ & 50 & $1: 100$ & 20 \\
\hline UM439(NW) & -1 & $1: 100$ & 10 & $1: 100$ & 10 \\
\hline UM439 & -0.861 & $1: 100$ & 10 & $1: 100$ & 10 \\
\hline UM448 & -0.866 & $1: 100$ & 50 & $1: 100$ & 50 \\
\hline UM448(extension NE-SW) & -0.704 & $1: 100$ & 20 & $1: 100$ & 20 \\
\hline UM456(NE) & -0.912 & $1: 100$ & 5 & $1: 100$ & 5 \\
\hline UM456(Cent) & -0.817 & $1: 100$ & 5 & $1: 100$ & 5 \\
\hline UM456(SW) & -0.923 & $1: 100$ & 5 & $1: 30$ & 10 \\
\hline UM456 & -0.922 & $1: 100$ & 1 & $1: 100$ & 10 \\
\hline UM461(E) & -1.068 & $1: 100$ & 1 & $1: 100$ & 1 \\
\hline UM461(W) & -1.330 & $1: 100$ & 5 & $1: 100$ & 2 \\
\hline UM461 & -1.101 & $1: 100$ & 5 & $1: 100$ & 2 \\
\hline UM462(SW) & -0.962 & $1: 30$ & 1 & $1: 30$ & 2 \\
\hline UM462(NE) & -0.914 & $1: 100$ & 5 & $1: 100$ & 2 \\
\hline UM462 & -0.926 & $1: 100$ & 5 & $1: 30$ & 1 \\
\hline UM477 & -1 & $1: 100$ & 509 & $1: 100$ & 500 \\
\hline UM483 & -1 & $1: 30$ & 50 & $1: 30$ & 50 \\
\hline UM499(W) & -1 & $1: 30$ & 102 & $1: 30$ & 50 \\
\hline UM533(E) & -0.503 & $1: 100$ & 20 & $1: 100$ & 20 \\
\hline UM533(W) & -1 & $1: 100$ & 2 & $1: 100$ & 1 \\
\hline UM533 & -0.598 & $1: 100$ & 10 & $1: 100$ & 10 \\
\hline UM598E(SW) & -1 & $1: 10$ & 509 & $1: 10$ & 500 \\
\hline UM598W(SW) & -1 & $1: 100$ & 20 & $1: 100$ & 20 \\
\hline UM598W(CentSW) & -1 & $1: 100$ & 102 & $1: 100$ & 50 \\
\hline UM598W(NE) & -1 & $1: 100$ & 50 & $1: 100$ & 50 \\
\hline IIZw40 & -0.796 & $1: 100$ & 2 & $1: 100$ & 2 \\
\hline
\end{tabular}

